\title{
28. MIOCENE POLLEN STRATIGRAPHY OF LEG 127 IN THE JAPAN SEA AND COMPARISON WITH THE STANDARD NEOGENE POLLEN FLORAS OF NORTHEAST JAPAN ${ }^{1}$
}

\author{
Tohru Yamanoi ${ }^{2}$
}

\begin{abstract}
Pollen floras were obtained from Miocene sediments recovered at four sites drilled during Ocean Drilling Program Leg 127. The local pollen floras of each site were correlated to the standard pollen zones of northeast Japan by using the concept of the essential members for each pollen zone. At Site 797, the complete floral range was obtained for recognition of the NP2 zone and the pollen components of the NP1 zone were also clarified continuously. The ages of the boundaries between pollen zones NP4/NP3, NP3/NP2, and NP2/NP1 are estimated to be about $7 \mathrm{Ma}, 13 \mathrm{Ma}$, and 17-18.5 Ma, respectively. Even in the same pollen zone, the ratios of major pollen taxa vary with the location. This variation is expressed on maps representing two different times during the Miocene.
\end{abstract}

\section{INTRODUCTION}

The Neogene pollen flora of northeast Japan has been summarized by this author (Yamanoi, 1989). However, the pollen floras of the early Miocene are not well known due to the lack of outcrops of this age in northeast Japan. During Ocean Drilling Program (ODP) Leg 127, drilling reached the lower Miocene sediments at all sites (794 through 797, Fig. 1). Examination for marine microfossils revealed that they occurred only a little, or were barren, in the lower Miocene sediments at each site (Tamaki, Pisciotto, Allan, et al., 1990). Therefore, pollen fossils were expected to determine the age for the lower Miocene sediments. Then, pollen analyses were carried out, with priority given to the lower samples of each hole. Though the analyses are continuing, the results obtained thus far are summarized as Miocene pollen flora.

One purpose of this study is to show whether or not the local pollen floras of each site can be correlated with the standard pollen zones of northeast Japan. The components of the essential members of specific pollen floras, plotted on ternary diagrams, are used as an effective means for the correlation of the floras. Moreover, it can be estimated that the allochthonous character of pollen grains is emphasized for their marine sediments. Therefore, how the allochthonous pollen sedimentation is expressed in different marine sites is studied.

\section{MATERIALS AND METHODS}

Approximately 100 samples of Miocene sediment were selected for pollen analyses from the four sites of Leg 127 (Fig. 1). The samples were dried and sieved through a screen ( 60 mesh) after powdering. All the samples were treated with $\mathrm{KOH}(10 \%), \mathrm{HF}(47 \%)$, and $\mathrm{HCl}$ $+\mathrm{HNO}_{3}(1: 1)$, then given acetolysis treatment, and finally treated with a solution of $\mathrm{ZnCl}_{2}$ (specific gravity of 2 ) to concentrate pollen grains. Slides were prepared for light microscope (LM) study by mounting pollen grains in glycerine jelly. The total pollen grains per gram of dry sediment was obtained by using a method of Traverse and Ginsburg (1966).

During the course of microscopic examination of each sample, 100 pollen grains were classified in order to obtain a Pinaceae (vesiculate type) pollen vs. other pollen ratio, and 200 pollen grains

\footnotetext{
' Pisciotto, K. A., Ingle, J. C., Jr., von Breymann, M. T., Barron, J., et al., 1992. Proc. ODP, Sci. Results, 127/128, Pt. 1: College Station, TX (Ocean Drilling Program).

${ }^{2}$ Department of Earth Sciences, Faculty of General Education, Yamagata University, Yamagata, 990 Japan.
}

were identified taxonomically, with the exception of vesiculate Pinaceae pollen.

\section{RESULTS OF POLLEN ANALYSES}

Pollen stratigraphic investigations for Leg 127 have been carried out in each hole in uphole sequence. The work is still continuing, but the results obtained so far are reported here. The pollen components and total pollen grains per gram of dry sediment of each sample are summarized in Table 1. Some occurrences of pollen fossils from each hole are briefly described here in north to south succession.

\section{Site 795}

This site is located in the northern Japan Sea, nearly equidistant from the coasts of Hokkaido and Sikhote-Aline, Siberia (Fig. 1). The results of pollen analyses for this site are summarized in a diagram showing major floral components (Fig. 2). The pollen flora of this site is characterized by a high percentage of Larix, Taxodiaceae, and Fagus in many samples. Especially, Taxodiaceae occurs very abundantly from Cores $127-795 \mathrm{~A}-34 \mathrm{X}$ to $127-795 \mathrm{~B}-1 \mathrm{R}$. In this zone, Alnus and Artemisia also have relative dominance, but in contrast, Larix is not abundant. Pterocarya, Alnus, Carpinus, D. (deciduous type) Quercus, and Ulmus are fairly abundant in almost all samples.

\section{Site 796}

This site is located on the Okushiri Ridge and not far from Hokkaido (Fig. 1). Major floral components of Hole 796B are shown in Figure 3. As can be seen this diagram, Taxodiaceae is dominant in many samples except for a few lower horizons. The percentage of $\mathrm{E}$. (evergreen type) Quercus becomes higher in lower horizons and reaches its maximum value $(48 \%)$ at the lowermost Sample 127 796B-32R-5, 60-62 cm. Alnus, Carpinus, Fagus, D. Quercus, and Artemisia are fairly abundant in many samples.

\section{Site 794}

This site is located in the northernmost Yamato Basin, approximately $175 \mathrm{~km}$ from the Oga Peninsula of northern Honshu (Fig. 1).

The major floral components of this site are shown in Figure 4. These flora are characterized by a high percentage of E. Quercus. D. Quercus is also fairly abundant in many horizons. The occurrence of Carya is high from Core 127-794B-19R downhole and Fagus occurrence is high from this core uphole. Larix is abundant locally with its highest value of $19 \%$ occurring at Sample 127-794A-30X-1, 130-132 cm. 
Table 1. List of pollen occurrences for each site.

\begin{tabular}{|c|c|c|c|c|c|c|c|c|c|c|c|c|c|}
\hline \multirow{2}{*}{$\begin{array}{c}\text { Hole: } \\
\text { Core, section: } \\
\text { Interval }(\mathrm{cm}):\end{array}$} & \multicolumn{3}{|c|}{$127-795 \mathrm{~A}-$} & \multicolumn{10}{|c|}{$127-795 \mathrm{~B}-$} \\
\hline & $\begin{array}{c}34 X-2 \\
135-136\end{array}$ & $\begin{array}{c}35 X-4 \\
135-137\end{array}$ & $\begin{array}{c}37 \mathrm{X}-1 \\
132-134\end{array}$ & $\begin{array}{c}1 R-1 \\
134-136\end{array}$ & $\begin{array}{l}8 \mathrm{R}-1 \\
54-56\end{array}$ & $\begin{array}{c}9 \mathrm{R}-2 \\
133-135\end{array}$ & $\begin{array}{l}10 R-2 \\
80-82\end{array}$ & $\begin{array}{l}\text { IIR-2 } \\
83-84\end{array}$ & $\begin{array}{l}12 R-2 \\
80-82\end{array}$ & $\begin{array}{l}13 R-2 \\
79-81\end{array}$ & $\begin{array}{l}14 R-2 \\
79-81\end{array}$ & $\begin{array}{l}15 R-2 \\
82-84\end{array}$ & $\begin{array}{c}16 \mathrm{R}-2 \\
122-124\end{array}$ \\
\hline \multicolumn{14}{|l|}{ Arboreal pollen } \\
\hline Larix & 0.5 & 1.0 & 2.5 & 1.5 & 18.5 & 29.0 & 0.5 & 23.0 & 21.0 & 17.0 & 8.5 & 11.5 & 5.0 \\
\hline Dacrydium & - & - & - & - & - & - & - & - & - & - & - & - & - \\
\hline Metasequoia & - & 2.0 & 2.0 & 3.0 & 0.5 & 0.5 & 0.5 & - & 1.0 & 0.5 & 2.0 & 1.5 & 1.5 \\
\hline Taxodiaceae & 42.5 & 32.0 & 43.0 & 31.5 & 15.5 & 14.5 & 38.5 & 13.5 & 20.0 & 2.5 & 11.0 & 12.0 & 11.5 \\
\hline Sciadopitys & - & - & - & - & - & - & - & - & - & - & - & - & - \\
\hline Cunninghamia & - & - & - & - & - & - & - & - & - & - & - & - & - \\
\hline Cupressaceae & - & - & - & - & 1.0 & 1.5 & - & - & 0.5 & 1.0 & 1.5 & - & - \\
\hline Ephedra & - & 0.5 & - & - & 1.0 & 1.0 & 0.5 & - & - & 0.5 & 0.5 & - & - \\
\hline Ginkgo & - & - & - & - & - & - & - & - & - & - & - & - & - \\
\hline Sarix & 0.5 & - & - & - & - & - & - & - & - & - & - & - & - \\
\hline Myrica & - & - & - & - & - & - & - & - & - & - & 0.5 & - & - \\
\hline Juglans & 0.5 & 0.5 & 0.5 & - & 1.0 & - & - & 0.5 & - & - & - & - & 1.5 \\
\hline Pterocarya & 4.0 & 4.0 & 3.0 & 3.0 & 7.0 & 6.0 & 5.5 & 5.5 & 17.0 & 1.5 & 4.0 & 5.5 & 9.0 \\
\hline Carya & 3.0 & 1.5 & 2.0 & 0.5 & 1.5 & 6.0 & 4.0 & 4.5 & 3.0 & 6.5 & 4.5 & 2.5 & 4.0 \\
\hline Engelhandia & - & 0.5 & - & 2.5 & 1.5 & 1.5 & 1.0 & 0.5 & - & 1.0 & 1.0 & 2.0 & 3.0 \\
\hline Alnus & 7.0 & 14.5 & 12.5 & 11.0 & 3.0 & 3.5 & 7.5 & 6.0 & 4.5 & 6.5 & 12.5 & 9.0 & 7.0 \\
\hline Betula & 4.5 & 4.5 & 1.5 & 2.0 & 3.5 & 1.0 & - & 3.0 & 4.0 & 0.5 & 2.0 & 3.0 & 2.0 \\
\hline Carpinus & 1.0 & 3.0 & 4.0 & 1.5 & 11.0 & 6.0 & 5.5 & 3.0 & 8.0 & 14.5 & 7.0 & 7.0 & 8.0 \\
\hline Corylus & 3.0 & 3.0 & 1.0 & 1.0 & 2.0 & - & 1.5 & 3.5 & 3.5 & 1.5 & 1.0 & 2.0 & 1.5 \\
\hline Castanea & - & - & - & - & 0.5 & 2.0 & 2.5 & 1.0 & 1.0 & 0.5 & - & 0.5 & 1.5 \\
\hline Fagus & 15.5 & 21.0 & 9.0 & 18.0 & 11.5 & 8.5 & 12.5 & 14.5 & 4.0 & 22.0 & 21.5 & 21.5 & 21.0 \\
\hline Pasania & - & - & - & - & 0.5 & - & - & 1.0 & 0.5 & - & 1.0 & 0.5 & 1.0 \\
\hline E. Quercus & - & 1.0 & - & - & 1.5 & 2.0 & 4.5 & 1.0 & 1.0 & 2.0 & 0.5 & 4.0 & 4.5 \\
\hline D. Quercus & 2.0 & 2.0 & 1.5 & 5.0 & 6.5 & 6.5 & 8.0 & 8.0 & 4.0 & 11.0 & 11.5 & 10.5 & 8.5 \\
\hline Ulmus & 1.0 & 2.0 & 2.5 & 3.5 & 8.0 & 6.5 & 4.5 & 4.5 & 3.0 & 6.5 & 4.0 & 3.0 & 3.5 \\
\hline Zelkova & 2.0 & 0.5 & 0.5 & 3.0 & 0.5 & 1.5 & 1.5 & 1.0 & - & 1.5 & 0.5 & 1.0 & 4.0 \\
\hline Celtys & - & - & - & - & - & - & - & - & - & - & - & - & - \\
\hline Eurya & - & - & - & - & - & - & - & - & - & - & - & - & - \\
\hline Liquidambar & - & - & 0.5 & 0.5 & 0.5 & 0.5 & 1.0 & - & - & - & - & - & 0.5 \\
\hline Sapium & - & - & - & - & - & - & - & - & - & - & - & - & - \\
\hline Rhus & - & - & 0.5 & - & - & - & - & - & - & - & - & - & - \\
\hline Acer & 0.5 & 0.5 & 1.0 & - & 1.0 & 0.5 & 0.5 & - & - & - & - & 1.0 & - \\
\hline Aesculus & 0.5 & - & - & - & - & - & - & - & - & - & - & - & 0.5 \\
\hline Hex & - & - & - & - & - & - & - & - & - & - & - & - & - \\
\hline Tilia & 1.5 & 0.5 & - & 2.0 & 1.5 & 1.5 & - & 2.0 & 1.0 & 1.0 & 1.0 & 0.5 & - \\
\hline Elaeagnus & - & - & - & - & - & - & - & - & - & - & - & - & - \\
\hline Lagerstroemia & - & - & - & - & - & - & - & - & - & - & - & - & - \\
\hline Alangium & - & - & - & - & - & - & - & - & - & - & - & - & - \\
\hline Nyssa & - & - & - & - & - & - & - & - & - & - & - & - & - \\
\hline Ericaceae & - & - & - & - & - & - & - & - & 1.0 & 0.5 & 3.0 & - & - \\
\hline Symplocos & - & - & - & - & - & - & - & - & - & - & - & - & - \\
\hline Ligustrum & - & - & - & - & - & - & - & - & - & - & - & - & - \\
\hline Weigela & - & - & - & - & - & - & - & - & - & 0.5 & - & - & - \\
\hline \multicolumn{14}{|l|}{ Non-arboreal pollen } \\
\hline Persicaria & - & - & - & - & - & - & - & - & - & - & - & 0.5 & - \\
\hline Chenopodiaceac & 1.0 & 0.5 & $\overline{1.0}$ & 1.0 & 0.5 & $\overline{-}$ & - & 0.5 & - & 0.5 & 1.0 & 0.5 & 0.5 \\
\hline Caryophyllaceae & - & - & 1.0 & - & - & - & - & - & - & - & - & - & - \\
\hline Ranunculaceae & - & - & - & - & - & - & - & - & - & - & - & - & - \\
\hline Trapa & - & - & - & - & - & - & - & - & - & - & - & - & - \\
\hline Patrinia & - & - & - & - & - & - & - & - & - & - & - & - & - \\
\hline Artemisia & 9.0 & 3.5 & 9.5 & 9.0 & 0.5 & - & - & 2.0 & 2.0 & - & - & - & - \\
\hline Carduoideae & 0.5 & - & 0.5 & 0.5 & - & - & - & 0.5 & - & 0.5 & - & - & - \\
\hline Cichorioideac & - & - & 0.5 & - & - & - & - & - & - & - & - & - & 0.5 \\
\hline Gramineae & - & 1.0 & - & - & - & - & - & 1.0 & - & - & - & 0.5 & - \\
\hline Sparganium & - & 0.5 & - & - & - & - & - & - & - & - & - & - & - \\
\hline Cyperaceae & - & - & - & - & - & - & - & - & - & - & - & - & - \\
\hline Fupingopollenites & - & - & - & - & - & - & - & - & - & - & - & - & - \\
\hline \multicolumn{14}{|l|}{ (Total $=100.0 \%$ ) } \\
\hline \multicolumn{14}{|c|}{ Assemblage of 100 grains } \\
\hline Abies & 3 & 2 & 1 & 4 & - & 4 & 3 & 2 & 3 & 3 & - & - & - \\
\hline Picea & 35 & $5 i$ & 45 & 30 & 50 & 61 & 16 & 54 & 50 & 38 & 42 & 27 & 29 \\
\hline Pinus & 21 & 13 & 14 & 28 & 22 & 9 & 13 & 18 & 20 & 19 & 11 & 23 & 15 \\
\hline Tsuga & 6 & 15 & 8 & 12 & 11 & 20 & 14 & II & 8 & 28 & 28 & 27 & 16 \\
\hline Others & 35 & 19 & 32 & 26 & 17 & 6 & 54 & 15 & 19 & 12 & 19 & 23 & 40 \\
\hline $\begin{array}{l}\text { Total pollen grains } \\
\text { per gram of sediment }\end{array}$ & 6240 & 3030 & 4150 & 15,040 & 5200 & 9160 & 2500 & 5710 & 11,390 & 5090 & 2550 & 4570 & 8600 \\
\hline
\end{tabular}


Table 1 (continued).

\begin{tabular}{|c|c|c|c|c|c|c|c|c|c|c|c|c|c|c|c|}
\hline \multicolumn{16}{|c|}{$127-795 \mathrm{~B}-$} \\
\hline $\begin{array}{c}17 R-1 \\
138-140\end{array}$ & $\begin{array}{c}18 R-2 \\
134-136\end{array}$ & $\begin{array}{c}19 R-2 \\
134-135\end{array}$ & $\begin{array}{c}20 \mathrm{R}-1 \\
131-133\end{array}$ & $\begin{array}{c}21 R-2 \\
132-134\end{array}$ & $\begin{array}{l}22 R-3 \\
50-52\end{array}$ & $\begin{array}{l}23 \mathrm{R}-3 \\
49-51\end{array}$ & $\begin{array}{l}24 R-4 \\
39-41\end{array}$ & 25R-5 & $\begin{array}{l}26 R-4 \\
88-90\end{array}$ & $\begin{array}{r}27 \mathrm{R}-2 \\
109-1\end{array}$ & $28 \mathrm{R}-3$ & $\begin{array}{c}29 \mathrm{R}-3 \\
\end{array}$ & $30 \mathrm{R}-3$ & $\begin{array}{c}31 R-3 \\
126-128\end{array}$ & $\begin{array}{c}32 \mathrm{R}-3 \\
125-127\end{array}$ \\
\hline & & & & & & & & & & & & & & & \\
\hline- & 8.5 & 5.5 & 0.5 & 11.0 & 11.5 & 10.0 & 4.0 & 14.5 & - & 9.5 & 8.5 & 3.5 & 8.0 & 4.5 & 6.0 \\
\hline 1.5 & - & - & - & - & - & - & - & - & 5.0 & - & - & - & - & - & - \\
\hline 2.5 & 0.5 & 4.0 & 2.0 & 0.5 & 2.0 & 5.5 & 2.5 & 1.0 & 1.5 & 5.0 & 1.0 & 1.5 & 2.0 & - & 1.0 \\
\hline 14.0 & 17.5 & 16.5 & 27.5 & 11.0 & 15.5 & 29.0 & 24.5 & 13.0 & 14.5 & 7.0 & 9.0 & 11.5 & 8.0 & 11.0 & 0.5 \\
\hline - & - & - & - & - & - & - & - & - & - & - & - & - & - & - & - \\
\hline- & - & - & - & - & - & - & - & - & - & - & - & - & - & - & - \\
\hline- & 1.0 & 0.5 & - & 1.0 & 2.0 & 0.5 & 0.5 & - & 1.0 & 1.5 & $\overline{1.0}$ & 1.0 & 1.0 & 2.0 & 0.5 \\
\hline 1.0 & 1.0 & - & - & 0.5 & - & 0.5 & - & - & - & 1.0 & 1.5 & 1.0 & - & - & 0.5 \\
\hline - & 0.5 & - & - & - & - & - & - & - & - & - & - & - & - & - & - \\
\hline- & - & - & - & - & - & - & - & - & - & - & - & - & - & - & - \\
\hline - & 0.5 & - & - & - & 1.0 & - & - & - & - & - & - & - & - & - & - \\
\hline 1.5 & - & $\overline{-}$ & $=$ & $\overline{1.5}$ & 1.5 & $=$ & $\overline{0.5}$ & $=$ & $\overrightarrow{1.0}$ & $\overline{1.5}$ & $\overline{1.5}$ & $=$ & $\overline{-}$ & 0.5 & 0.5 \\
\hline 8.5 & 1.5 & 2.0 & 2.0 & 4.0 & 5.5 & 2.5 & 1.5 & 4.0 & 1.0 & 1.0 & 5.0 & 5.5 & 8.5 & 6.0 & 7.5 \\
\hline 8.0 & 3.0 & 4.5 & 2.0 & 3.0 & 6.5 & 3.0 & 3.5 & 5.5 & 4.0 & 5.5 & 8.0 & 5.0 & 6.0 & 10.0 & 8.5 \\
\hline 1.0 & 0.5 & 1.5 & 1.5 & 2.0 & 1.5 & 5.0 & 0.5 & 4.0 & 3.5 & 4.5 & 3.5 & 2.0 & 2.0 & 10.0 & 2.5 \\
\hline 6.0 & 10.0 & 8.0 & 7.0 & 6.5 & 4.0 & 6.0 & 7.5 & 9.0 & - & 4.0 & 9.0 & 6.5 & 7.5 & 3.5 & 3.0 \\
\hline 1.5 & 3.5 & 4.0 & 3.5 & 3.0 & 3.0 & 1.5 & 0.5 & 1.5 & 5.5 & 1.5 & 4.0 & 2.5 & 3.0 & 5.0 & 1.0 \\
\hline 7.0 & 6.0 & 3.0 & 5.5 & 8.5 & 3.0 & 2.0 & 1.0 & 1.5 & 2.0 & 3.5 & 4.5 & 5.5 & 1.0 & 4.0 & 2.5 \\
\hline 1.5 & 0.5 & 1.0 & 1.0 & 0.5 & 0.5 & - & 2.0 & - & 0.5 & - & 0.5 & 1.5 & 1.5 & 0.5 & 3.0 \\
\hline- & 1.5 & 1.0 & - & 2.0 & - & - & 2.0 & - & - & 0.5 & - & 0.5 & 1.0 & 0.5 & - \\
\hline 18.5 & 25.0 & 29.5 & 29.5 & 16.5 & 22.5 & 18.5 & 24.5 & 34.5 & 42.0 & 38.0 & 27.0 & 33.5 & 35.0 & 28.0 & 43.0 \\
\hline 1.0 & - & 1.0 & - & 0.5 & 0.5 & - & 1.5 & - & 0.5 & - & 0.5 & - & 0.5 & - & - \\
\hline 6.5 & 3.5 & 6.0 & 4.5 & 7.5 & 6.0 & 2.0 & 7.0 & 2.0 & 2.5 & 2.0 & 1.5 & 3.5 & 3.5 & 3.5 & 5.0 \\
\hline 10.0 & 8.0 & 6.5 & 3.5 & 12.5 & 4.0 & 6.0 & 9.0 & 2.5 & 6.0 & 7.5 & 5.5 & 5.5 & 5.5 & 4.0 & 7.0 \\
\hline 6.5 & 3.0 & 3.0 & 4.5 & 5.0 & 5.5 & 4.5 & 4.5 & 4.0 & 6.0 & 1.5 & 4.5 & 3.0 & 2.0 & 4.0 & 2.0 \\
\hline- & 1.0 & - & 2.0 & 1.5 & 1.5 & 1.0 & 2.5 & - & 1.0 & 3.0 & 1.5 & 4.0 & 3.0 & 1.0 & 1.0 \\
\hline- & - & - & - & - & - & - & - & - & - & - & - & - & - & - & - \\
\hline- & - & - & - & - & - & - & - & - & - & - & - & - & - & - & - \\
\hline- & - & - & 0.5 & - & - & 0.5 & - & - & 0.5 & 0.5 & - & 0.5 & 1.0 & - & 1.5 \\
\hline- & - & - & - & - & - & - & - & - & - & - & - & - & - & - & - \\
\hline- & - & - & - & - & - & - & - & - & - & - & - & - & - & - & - \\
\hline 0.5 & 1.0 & 0.5 & 0.5 & 0.5 & 0.5 & 0.5 & - & 1.0 & - & 0.5 & 2.0 & 0.5 & - & - & 0.5 \\
\hline- & - & - & - & - & - & - & - & - & - & - & - & - & - & - & - \\
\hline- & - & - & - & - & - & - & - & - & - & - & - & - & - & - & - \\
\hline 1.5 & 0.5 & 0.5 & 0.5 & 1.0 & 0.5 & 1.0 & - & 1.0 & 1.0 & 0.5 & - & 2.0 & $\overline{-}$ & 1.0 & 0.5 \\
\hline- & 0.5 & - & - & - & - & - & - & - & - & - & - & - & - & - & - \\
\hline- & - & - & - & $\overline{-}$ & - & - & - & $\overline{-}$ & $\overline{-}$ & - & - & - & - & - & - \\
\hline- & - & - & - & - & - & - & - & - & - & - & - & - & - & - & - \\
\hline- & - & - & - & - & - & - & - & - & - & - & - & - & - & - & - \\
\hline 1.0 & 0.5 & 1.0 & 1.0 & - & 1.0 & - & 0.5 & 0.5 & - & - & 0.5 & - & - & 1.0 & 1.5 \\
\hline- & - & - & - & - & - & - & - & - & - & - & - & - & - & - & - \\
\hline- & - & - & - & - & - & - & - & - & - & - & - & - & - & - & - \\
\hline- & 0.5 & - & - & - & - & - & - & - & - & - & - & - & - & - & - \\
\hline- & - & - & - & - & - & - & - & - & - & - & - & - & - & - & - \\
\hline- & 0.5 & - & 1.0 & $=$ & $=$ & $\overline{0.5}$ & $\overline{-}$ & $\overline{-}$ & $\bar{z}$ & - & - & - & - & - & - \\
\hline- & - & - & - & - & - & - & - & - & - & - & - & - & - & - & - \\
\hline- & - & - & - & - & - & - & - & - & - & - & - & - & - & - & $\overline{1}$ \\
\hline- & - & - & - & - & - & - & - & 0.5 & - & - & - & - & - & - & 1.0 \\
\hline- & - & - & - & - & - & - & - & - & - & - & - & - & - & - & - \\
\hline- & - & - & - & - & - & - & - & - & - & - & - & - & - & - & - \\
\hline- & - & - & - & - & - & - & - & - & - & - & - & - & - & - & - \\
\hline 0.5 & - & - & - & - & 0.5 & - & - & - & - & - & - & - & - & - & - \\
\hline- & - & - & - & - & - & - & - & - & 1.0 & - & - & - & - & - & - \\
\hline- & - & - & - & - & - & - & - & - & - & - & - & - & - & - & - \\
\hline$=$ & - & $=$ & $=$ & $=$ & $\overline{-}$ & $=$ & $=$ & $=$ & $\overline{-}$ & - & - & - & - & - & - \\
\hline- & - & 0.5 & - & - & - & - & - & - & $=$ & 0.5 & - & - & - & - & - \\
\hline 3 & 2 & 2 & 2 & - & 3 & 3 & - & - & - & - & - & - & 2 & - & - \\
\hline 35 & 52 & 49 & 22 & 34 & 47 & 39 & 28 & 30 & 61 & 33 & 36 & 43 & 26 & 46 & 51 \\
\hline 6 & 12 & 9 & 7 & 18 & 10 & 5 & 16 & 20 & 6 & 12 & 19 & 9 & 17 & 9 & 15 \\
\hline 23 & 19 & 27 & 17 & 19 & 31 & 40 & 19 & 27 & 28 & 24 & 26 & 36 & 24 & 22 & 18 \\
\hline 33 & 15 & 13 & 52 & 29 & 9 & 13 & 37 & 23 & 5 & 31 & 19 & 12 & 31 & 23 & 16 \\
\hline 11,110 & 8640 & 5820 & 940 & 4220 & 6020 & 11,570 & 2310 & 1340 & 4400 & 4890 & 3880 & 7250 & 10.190 & 6483 & 3930 \\
\hline
\end{tabular}


Table 1 (continued).

\begin{tabular}{|c|c|c|c|c|c|c|c|c|c|c|c|c|c|c|}
\hline \multirow{3}{*}{$\begin{array}{c}\text { Hole: } \\
\text { Core, section: } \\
\text { Interval }(\mathrm{cm}) \text { : }\end{array}$} & \multirow{3}{*}{ 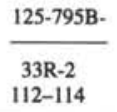 } & \multicolumn{13}{|c|}{ 127-796B- } \\
\hline & & 10R-1 & $11 \mathrm{R}-1$ & $12 \mathrm{R}-2$ & 14R-1 & $15 R-2$ & $17 \mathrm{R}-\mathrm{CC}$ & $18 R-1$ & 19R-1 & 20R-1 & 2IR-1 & $22 \mathrm{R}-1$ & $26 \mathrm{R}-1$ & $27 R-1$ \\
\hline & & $68-70$ & $108-110$ & 95-97 & $53-55$ & $42-44$ & $6-8$ & $123-125$ & $133-135$ & $29-31$ & $81-83$ & $62-64$ & $72-74$ & 98-100 \\
\hline \multicolumn{15}{|l|}{ Arboreal pollen } \\
\hline Larix & 14.0 & 1.0 & - & 3.0 & 2.0 & 1.0 & 2.5 & 4.0 & 2.0 & 4.5 & 2.5 & 1.0 & - & 2.5 \\
\hline Dacrydium & - & - & - & - & - & - & - & - & - & - & - & - & - & - \\
\hline Metasequoia & 1.5 & 4.5 & 2.0 & 2.0 & 5.5 & 6.0 & 8.0 & 2.5 & 0.5 & 3.0 & 1.5 & 6.0 & 1.5 & 1.5 \\
\hline Taxodiaceae & 2.0 & 22.5 & 38.5 & 32.5 & 32.0 & 36.5 & 41.5 & 33.0 & 30.5 & 24.5 & 19.0 & 24.5 & 48.5 & 46.0 \\
\hline Sciadopitys & - & - & - & 0.5 & 2.0 & 2.0 & - & 2.0 & 1.0 & - & - & - & - & - \\
\hline Cunninghamia & 0.5 & - & - & - & - & - & - & - & - & - & - & - & - & - \\
\hline Cupressaceae & - & - & - & - & 1.0 & 0.5 & - & - & - & - & - & - & - & - \\
\hline Ephedra & 0.5 & 1.0 & 0.5 & - & - & 1.5 & 1.0 & - & 1.5 & - & - & 1.0 & 0.5 & 1.0 \\
\hline Ginkgo & - & - & - & - & - & - & - & - & - & - & - & - & - & - \\
\hline Sarix & - & - & - & - & - & - & - & - & - & - & - & - & - & - \\
\hline Myrica & - & - & - & - & - & - & - & - & - & - & - & - & - & - \\
\hline Juglans & - & - & - & - & - & - & - & - & - & 0.5 & 1.5 & - & - & - \\
\hline Pterocarya & 3.5 & 3.0 & 4.5 & 1.5 & 5.5 & 3.5 & 3.5 & 2.0 & 2.0 & 3.0 & 5.5 & 3.0 & 4.5 & 2.0 \\
\hline Carya & 7.0 & 0.5 & 0.5 & 3.0 & 5.0 & 2.5 & 1.0 & 1.0 & 5.5 & 9.0 & 9.0 & 4.5 & 3.0 & 3.0 \\
\hline Engelhardia & 1.5 & - & 0.5 & 1.0 & 1.0 & - & 1.5 & 0.5 & 0.5 & 1.0 & 0.5 & 2.0 & - & 1.5 \\
\hline Alnus & 1.5 & 13.5 & 10.5 & 11.0 & 6.5 & 4.5 & 4.0 & 11.5 & 3.0 & 3.5 & 4.5 & 6.0 & 5.5 & 6.0 \\
\hline Betula & 2.5 & 2.0 & 1.5 & 3.5 & 2.5 & 3.0 & 3.5 & 4.0 & 4.5 & 3.5 & 3.5 & - & 4.0 & 1.5 \\
\hline Carpinus & 4.0 & 6.5 & 5.5 & 4.0 & 4.0 & 6.0 & 2.5 & 6.0 & 4.0 & 6.0 & 4.5 & 6.5 & 2.5 & 3.0 \\
\hline Corylus & 0.5 & 1.5 & 1.5 & 2.5 & 1.5 & 1.0 & 1.0 & 2.5 & 1.5 & 1.5 & 2.0 & 3.5 & 2.0 & 0.5 \\
\hline Castanea & 0.5 & 0.5 & 0.5 & - & - & - & 0.5 & - & 3.0 & 0.5 & 1.0 & 0.5 & - & 0.5 \\
\hline Fagus & 32.0 & 19.5 & 16.5 & 16.0 & 7.0 & 6.5 & 9.0 & 14.0 & 10.5 & 9.0 & 10.0 & 11.5 & 9.5 & 4.5 \\
\hline Pasania & 4.5 & - & - & - & 1.0 & - & - & - & 3.5 & 0.5 & 2.5 & - & 1.0 & 0.5 \\
\hline E. Quercus & 9.0 & 4.0 & 1.5 & 0.5 & 3.5 & 3.0 & 3.5 & 4.0 & 7.5 & 9.5 & 6.0 & 7.5 & 4.0 & 6.0 \\
\hline D. Quercus & 6.5 & 2.0 & 4.5 & 3.0 & 4.5 & 4.5 & 3.5 & 2.5 & 4.5 & 5.0 & 10.5 & 8.5 & 7.0 & 6.0 \\
\hline Ulmus & 2.5 & 1.0 & 0.5 & 2.5 & 2.0 & - & 2.0 & 0.5 & 2.0 & 2.5 & 1.0 & 4.0 & 1.5 & 3.0 \\
\hline Zelkova & 2.0 & 5.0 & 1.5 & - & 2.0 & 2.5 & 1.0 & 2.0 & 2.0 & 3.5 & 2.0 & 1.0 & 1.0 & 2.0 \\
\hline Celtys & - & - & - & - & - & - & - & - & - & - & - & - & - & - \\
\hline Eurya & - & - & - & - & - & - & - & - & - & - & - & - & - & - \\
\hline Liquidambar & 2.0 & - & - & 2.5 & 0.5 & - & 0.5 & 0.5 & 0.5 & 2.0 & 0.5 & - & - & - \\
\hline Sapium & - & - & - & - & - & - & - & - & - & - & - & - & - & - \\
\hline Rhus & - & - & - & - & - & - & - & - & - & - & - & - & - & - \\
\hline Acer & 1.5 & - & 0.5 & - & - & 0.5 & - & 0.5 & 1.0 & - & - & - & - & - \\
\hline Aesculus & - & 0.5 & - & - & - & - & - & - & 0.5 & - & - & 0.5 & - & 0.5 \\
\hline Hex & - & - & - & - & - & - & - & 0.5 & - & - & - & - & - & - \\
\hline Tilia & 0.5 & 1.5 & 1.5 & 1.5 & 2.5 & 2.0 & 0.5 & 2.0 & 2.0 & - & 3.5 & 1.0 & 0.5 & 0.5 \\
\hline Elaeagnus & - & - & - & - & - & - & - & - & - & - & - & - & - & - \\
\hline Lagerstroemia & - & - & - & - & - & - & - & - & - & - & - & - & - & - \\
\hline Alangium & - & - & - & - & - & - & - & - & - & - & - & - & - & - \\
\hline Nyssa & - & - & 0.5 & - & 1.0 & - & 0.5 & - & - & - & - & - & - & 0.5 \\
\hline Ericaceae & - & - & 1.0 & - & - & - & - & - & - & 0.5 & - & - & - & 0.5 \\
\hline Symplocos & - & - & - & - & - & - & - & - & - & - & - & - & - & - \\
\hline Ligustrum & - & - & - & - & - & - & - & - & - & - & - & - & - & - \\
\hline Weigela & - & 0.5 & - & 0.5 & - & - & - & - & - & - & - & - & - & - \\
\hline \multicolumn{15}{|l|}{ Non-arboreal pollen } \\
\hline Persicaria & - & 0.5 & 0.5 & - & - & - & - & - & 1.0 & - & - & - & - & - \\
\hline Chenopodiaceae & - & 1.0 & 0.5 & 1.0 & 1.5 & 2.5 & 1.0 & 0.5 & 1.5 & 1.0 & 3.0 & 4.0 & 1.0 & 1.5 \\
\hline Caryophyllaceae & - & - & - & - & - & - & - & - & - & - & - & - & - & - \\
\hline Ranunculaceac & - & - & - & - & - & - & - & - & - & - & - & - & - & - \\
\hline Trapa & - & - & - & - & 0.5 & - & - & 0.5 & - & - & - & - & - & - \\
\hline Patrinia & - & - & - & - & - & 0.5 & - & - & - & - & - & - & - & - \\
\hline Artemisia & - & 7.5 & 4.0 & 6.0 & 4.0 & 8.0 & 6.5 & 3.0 & 3.5 & 5.5 & 6.0 & 3.5 & 2.5 & 4.5 \\
\hline Carduoideae & - & - & - & 1.5 & - & 2.0 & 1.0 & 0.5 & 0.5 & 0.5 & - & - & - & - \\
\hline Cichorioideae & - & - & - & - & 1.5 & - & - & - & - & - & - & - & - & - \\
\hline Gramineac & - & 0.5 & 1.0 & - & - & - & 0.5 & - & - & - & - & - & - & 1.0 \\
\hline Sparganium & - & - & - & - & - & - & - & - & - & - & - & - & - & - \\
\hline Cyperaceac & - & - & - & 0.5 & - & - & - & - & - & - & - & - & - & - \\
\hline Fupingopollenites & - & - & - & - & - & - & - & - & - & - & - & - & - & - \\
\hline
\end{tabular}

(Total $=100.0 \%$ )

Assemblage of 100 grains

\begin{tabular}{|c|c|c|c|c|c|c|c|c|c|c|c|c|c|c|}
\hline Abies & - & - & 1 & - & - & - & - & - & - & - & - & - & - & - \\
\hline Picea & 44 & 59 & 59 & 47 & 52 & 58 & 70 & 60 & 45 & 60 & 43 & 51 & 56 & 39 \\
\hline Pinus & 12 & 9 & 8 & 27 & 23 & 17 & 8 & 19 & 17 & 12 & 19 & 8 & 14 & 8 \\
\hline Tsuga & 29 & 12 & 15 & 10 & 11 & 6 & 9 & 9 & 11 & 15 & 18 & 18 & 5 & 12 \\
\hline Others & 15 & 20 & 17 & 16 & 14 & 19 & 13 & 12 & 27 & 13 & 20 & 23 & 25 & 41 \\
\hline $\begin{array}{l}\text { Total pollen grains } \\
\text { per gram of sediment }\end{array}$ & 8070 & 1450 & 3740 & 3070 & 3020 & 6740 & 7170 & 3310 & 8200 & 10,070 & 7780 & 2270 & 14,810 & 10,490 \\
\hline
\end{tabular}


Table 1 (continued).

\begin{tabular}{|c|c|c|c|c|c|c|c|c|c|c|c|c|c|c|c|}
\hline \multicolumn{5}{|c|}{ 127-796B- } & \multicolumn{11}{|c|}{$127-794 \mathrm{~A}-$} \\
\hline 28R-1 & $29 \mathrm{R}-1$ & 30R-1 & 3IR-2 & $32 R-5$ & $29 \mathrm{X}-1$ & $30 \mathrm{X}-1$ & $31 X-2$ & $32 X-3$ & $33 \mathrm{X}-3$ & $35 X-3$ & $36 \mathrm{X}-2$ & $37 X-2$ & $9 \mathrm{R}-1$ & $12 R-2$ & 13R-2 \\
\hline $76-78$ & $80-81$ & $83-85$ & $60-62$ & $60-62$ & $130-132$ & $130-132$ & $130-132$ & $130-132$ & $130-132$ & $117-119$ & $39-41$ & $127-129$ & $74-76$ & $116-118$ & 73-75 \\
\hline 0.5 & 3.5 & 2.0 & 2.0 & 2.5 & 1.5 & 19.0 & 7.0 & 7.0 & 2.0 & 2.0 & 3.0 & - & - & 0.5 & 1.5 \\
\hline$\overline{s 0}$ & $\bar{c}$ & - & - & - & - & - & - & - & 0.5 & - & - & - & - & 0.5 & - \\
\hline 5.0 & 4.5 & 4.0 & 4.5 & 1.5 & 1.0 & 3.5 & 2.0 & - & 5.0 & 0.5 & 1.5 & 7.5 & 4.0 & 1.5 & - \\
\hline 37.5 & 12.0 & 10.5 & 6.0 & 4.5 & 5.0 & 4.0 & 7.0 & 2.5 & 9.5 & 2.0 & - & 7.5 & 10.5 & 7.0 & 3.0 \\
\hline- & - & - & - & - & - & - & - & - & - & - & - & - & - & - & - \\
\hline - & - & - & - & - & - & - & - & - & - & - & - & - & - & - & - \\
\hline- & - & - & - & - & - & - & - & - & - & 0.5 & - & - & - & 1.0 & 1.0 \\
\hline - & 1.0 & 1.0 & 3.5 & - & 0.5 & - & 0.5 & - & 0.5 & - & 0.5 & 2.0 & 1.5 & - & 0.5 \\
\hline- & - & - & - & - & - & - & - & - & - & - & - & - & - & - & - \\
\hline- & - & - & - & - & - & - & - & - & - & - & - & - & - & - & - \\
\hline - & - & - & - & 0.5 & - & - & 0.5 & - & - & - & - & - & - & - & - \\
\hline- & - & 3.0 & - & - & - & - & 0.5 & - & - & 1.0 & - & - & - & 0.5 & - \\
\hline 2.0 & 7.5 & 9.0 & 5.5 & 2.0 & 7.0 & 15.0 & 4.5 & 9.0 & 0.5 & 5.5 & 4.5 & 4.5 & 5.5 & 5.5 & 5.0 \\
\hline 3.5 & 7.0 & 12.0 & 13.5 & 11.0 & 7.5 & 15.5 & 9.0 & 6.5 & 6.0 & 18.0 & 5.0 & 6.0 & 10.5 & 2.5 & 6.0 \\
\hline 1.0 & 0.5 & 4.0 & 1.0 & 1.0 & - & - & - & 0.5 & 0.5 & 0.5 & 0.5 & 2.0 & - & - & 1.0 \\
\hline 7.0 & 5.0 & 6.0 & 1.5 & 0.5 & 2.5 & 3.0 & 2.0 & 1.5 & 3.0 & 0.5 & - & 1.5 & 3.5 & 1.0 & 1.5 \\
\hline 2.0 & 2.0 & 1.0 & 3.0 & 1.5 & 2.5 & 1.0 & 5.0 & 2.5 & 1.0 & 3.0 & 1.5 & 2.0 & 4.0 & 3.0 & 1.0 \\
\hline 4.0 & 7.5 & 6.5 & 3.0 & 3.5 & 2.5 & 1.0 & 0.5 & 7.5 & 9.0 & 9.5 & 6.0 & 3.0 & 3.5 & 3.5 & 2.0 \\
\hline 1.5 & 1.5 & 0.5 & - & - & 0.5 & 4.0 & 1.5 & - & - & 1.5 & - & - & 0.5 & 0.5 & - \\
\hline 0.5 & 2.0 & - & 0.5 & - & 1.5 & - & 2.5 & 2.0 & 2.5 & 0.5 & 3.0 & 4.0 & 0.5 & 4.0 & 3.0 \\
\hline 8.0 & 9.5 & 9.5 & 3.5 & 8.5 & 10.5 & 6.5 & 11.0 & 19.5 & 13.0 & 19.5 & 15.0 & 8.0 & 12.5 & 20.5 & 24.5 \\
\hline 2.0 & 3.5 & 0.5 & 2.0 & 3.5 & 11.0 & - & 3.5 & 0.5 & 1.5 & 0.5 & 4.5 & 4.0 & 3.5 & 9.5 & 5.5 \\
\hline 9.5 & 9.5 & 5.0 & 20.5 & 48.0 & 5.5 & 2.0 & 9.5 & 11.0 & 22.5 & 14.5 & 38.0 & 30.0 & 24.5 & 18.5 & 28.0 \\
\hline 6.0 & 8.0 & 5.0 & 6.5 & 7.0 & 22.5 & 10.0 & 16.5 & 14.0 & 11.5 & 8.5 & 9.5 & 11.5 & 5.0 & 9.0 & 7.0 \\
\hline 1.0 & 4.5 & 9.0 & 2.0 & 0.5 & 3.0 & 2.5 & 3.5 & 5.5 & 4.5 & 6.0 & 3.5 & 1.5 & 3.0 & 4.5 & 2.0 \\
\hline 0.5 & 4.0 & 6.0 & 0.5 & 2.5 & 3.0 & 5.5 & 4.0 & 2.0 & 2.0 & 2.0 & 1.0 & 2.5 & 3.0 & 2.5 & 1.5 \\
\hline- & - & - & - & - & - & - & - & - & - & - & - & - & 1.0 & - & - \\
\hline- & - & - & 0.5 & - & - & - & - & - & - & - & - & - & - & - & $\overline{30}$ \\
\hline- & 0.5 & - & 0.5 & - & 2.0 & 1.5 & 1.0 & 1.0 & - & 2.5 & - & - & 0.5 & 2.0 & 3.0 \\
\hline- & - & - & - & - & - & - & - & - & - & - & - & - & - & - & - \\
\hline- & - & - & - & 0.5 & - & - & - & 0.5 & - & - & - & - & - & - & - \\
\hline- & 0.5 & - & - & - & - & - & 1.0 & 2.5 & - & - & - & - & 1.0 & 1.5 & 0.5 \\
\hline- & - & 0.5 & - & - & - & - & - & 0.5 & - & 0.5 & - & - & - & - & - \\
\hline- & - & - & - & - & - & - & - & - & - & - & - & - & - & - & $\bar{c}$ \\
\hline 0.5 & - & 3.5 & 0.5 & 0.5 & 1.5 & 1.0 & 2.0 & 0.5 & - & 1.0 & 2.5 & 1.0 & - & 0.5 & 1.5 \\
\hline- & - & - & - & - & - & - & - & - & - & - & - & - & - & - & - \\
\hline- & - & - & - & - & - & - & - & - & - & - & - & - & - & - & - \\
\hline- & - & - & - & - & - & - & - & - & - & - & - & - & - & - & - \\
\hline- & - & - & - & - & - & - & - & - & - & - & - & - & - & - & - \\
\hline- & - & - & 0.5 & 0.5 & - & - & - & - & - & - & - & - & - & - & 0.5 \\
\hline- & - & - & - & - & - & - & - & - & - & - & - & - & - & - & - \\
\hline- & - & - & - & - & - & - & - & - & - & - & - & - & - & - & - \\
\hline- & - & - & 1.0 & - & - & - & - & - & - & - & - & - & - & - & - \\
\hline- & - & - & - & - & - & - & - & - & - & - & - & 0.5 & - & - & - \\
\hline 3.5 & 2.0 & 1.0 & 14.5 & - & 3.0 & 1.0 & 1.0 & 2.0 & 3.5 & - & 0.5 & 1.0 & 2.0 & 0.5 & - \\
\hline- & - & - & - & - & - & - & - & - & - & - & - & - & - & - & - \\
\hline- & - & - & - & - & - & - & - & - & - & - & - & - & - & - & - \\
\hline- & - & - & - & - & - & - & - & - & - & - & - & - & - & - & - \\
\hline- & - & - & - & - & - & - & - & - & - & - & - & - & - & - & - \\
\hline 4.0 & 4.0 & - & 1.5 & - & 4.0 & 2.5 & 1.0 & 0.5 & - & - & - & - & - & - & - \\
\hline 0.5 & - & - & 2.0 & - & 1.0 & - & - & 0.5 & 0.5 & - & - & - & - & - & - \\
\hline- & - & - & - & - & 0.5 & 1.0 & - & 0.5 & - & - & - & - & - & - & $\overline{0.5}$ \\
\hline- & - & - & - & - & 0.5 & - & 1.0 & - & 1.0 & - & - & - & - & - & 0.5 \\
\hline- & - & - & - & - & - & - & 1.0 & - & - & - & - & - & - & - & - \\
\hline- & - & 0.5 & - & - & - & 0.5 & 1.5 & - & - & - & - & - & - & - & - \\
\hline- & - & - & - & - & - & - & - & - & - & - & - & - & - & - & - \\
\hline
\end{tabular}

\begin{tabular}{|c|c|c|c|c|c|c|c|c|c|c|c|c|c|c|c|}
\hline- & - & - & - & 2 & 3 & 4 & 9 & 5 & 2 & 3 & 2 & - & - & - & - \\
\hline 30 & 57 & 44 & 56 & 57 & 42 & 54 & 48 & 28 & 40 & 20 & 24 & 46 & 46 & 20 & 10 \\
\hline 15 & 22 & 38 & 28 & 15 & 9 & 8 & 10 & 12 & 24 & 17 & 23 & 18 & 15 & 26 & 24 \\
\hline 9 & 11 & 9 & 3 & 9 & 16 & 20 & 14 & 10 & 17 & 13 & 19 & 18 & 9 & 5 & 3 \\
\hline 46 & 10 & 9 & 13 & 17 & 30 & 14 & 19 & 45 & 17 & 47 & 32 & 18 & 30 & 49 & 63 \\
\hline 8240 & 8394 & 2020 & 11,800 & 12,530 & 19,240 & 9340 & 8760 & 3970 & 9170 & 54,120 & 46,850 & 1350 & 2950 & 8080 & 4870 \\
\hline
\end{tabular}


Table 1 (continued).

\begin{tabular}{|c|c|c|c|c|c|c|c|c|c|c|c|c|c|}
\hline \multirow{3}{*}{$\begin{array}{c}\text { Hole: } \\
\text { Core, section: } \\
\text { Interval }(\mathrm{cm}) \text { : }\end{array}$} & \multicolumn{7}{|c|}{$127-794 \mathrm{~A}-$} & \multicolumn{6}{|c|}{ 127-797B- } \\
\hline & $14 \mathrm{R}-2$ & 15R-2 & 19R-1 & 20R-1 & $21 \mathrm{R}-1$ & $24 \mathrm{R}-1$ & 25R-CC & $33 \mathrm{X}-3$ & $34 X-5$ & $35 \mathrm{X}-2$ & $37 \mathrm{X}-2$ & $38 \mathrm{X}-2$ & $39 \mathrm{X}-1$ \\
\hline & $71-72$ & $30-31$ & 69-71 & $115-117$ & $113-115$ & $21-23$ & $13-15$ & $40-42$ & $40-42$ & $39-41$ & $40-42$ & $18-20$ & $17-19$ \\
\hline Arboreal pollen & & & & & & & & & & . & & & \\
\hline Larix & 1.0 & 0.5 & 1.5 & 6.0 & 9.0 & 2.0 & 2.0 & - & - & - & - & - & - \\
\hline Dacrydium & 0.5 & - & 0.5 & - & - & 0.5 & 3.5 & - & - & - & - & - & - \\
\hline Metasequoia & 2.0 & - & 3.0 & 5.0 & 5.5 & 0.5 & 2.5 & - & - & - & - & - & - \\
\hline Taxodiaceae & 3.0 & 3.0 & 1.5 & 6.0 & 3.0 & 0.5 & 1.5 & 2.0 & 1.5 & 2.5 & 2.0 & 2.5 & 7.0 \\
\hline Sciadopitys & - & - & - & - & - & - & - & - & - & - & - & - & - \\
\hline Cunninghamia & - & - & - & - & - & - & - & - & - & - & - & - & - \\
\hline Cupressaceae & - & 1.0 & 1.0 & - & - & - & - & - & - & 1.0 & - & - & 1.0 \\
\hline Ephedra & 1.0 & 0.5 & - & - & 0.5 & - & 0.5 & 0.5 & - & 0.5 & - & - & 0.5 \\
\hline Ginkgo & - & - & - & - & - & - & - & - & - & - & - & - & - \\
\hline Sarix & - & - & - & - & - & - & - & - & - & - & - & - & - \\
\hline Myrica & - & - & - & - & - & - & - & 0.5 & - & - & - & - & 1.0 \\
\hline Juglans & 1.5 & - & 0.5 & 1.0 & 1.0 & - & - & - & 0.5 & - & - & - & - \\
\hline Plerocarya & 4.0 & 0.5 & 5.0 & 8.5 & 13.5 & 16.5 & 5.0 & 6.5 & 3.5 & 4.0 & 4.0 & 5.0 & 6.5 \\
\hline Carya & 8.0 & 2.5 & 11.5 & 18.0 & 20.5 & 25.5 & 27.0 & 9.0 & 19.0 & 20.5 & 17.5 & 10.0 & 11.5 \\
\hline Engelhardia & - & - & - & - & - & - & - & - & 1.0 & - & - & - & - \\
\hline Alnus & 1.0 & 1.5 & 0.5 & - & 1.0 & - & 1.0 & 1.5 & 0.5 & - & 0.5 & 2.0 & 1.5 \\
\hline Betula & 2.0 & 2.5 & 0.5 & 1.0 & 2.0 & - & - & 1.5 & 2.5 & 1.0 & 0.5 & 1.5 & 4.5 \\
\hline Carpinus & 4.5 & 3.0 & 2.0 & 6.0 & 4.0 & 4.5 & 4.0 & 4.0 & 4.5 & 2.0 & 5.5 & 3.5 & 2.0 \\
\hline Corylus & - & - & 1.0 & - & 0.5 & - & 1.0 & 1.0 & 1.0 & 3.5 & 0.5 & 0.5 & 2.5 \\
\hline Castanea & 1.0 & 1.5 & 2.5 & 1.5 & 4.0 & 1.0 & 2.0 & 1.0 & 0.5 & 2.5 & 0.5 & - & 0.5 \\
\hline Fagus & 23.0 & 10.5 & 3.5 & 8.0 & 6.0 & 5.5 & 6.5 & 30.5 & 18.0 & 14.0 & 22.0 & 32.0 & 26.5 \\
\hline Pasania & 4.5 & 18.0 & 7.0 & 1.5 & 3.0 & 3.5 & 5.0 & 1.5 & 3.0 & 6.5 & 0.5 & 1.5 & 1.0 \\
\hline E. Quercus & 13.5 & 38.5 & 35.0 & 18.0 & 13.0 & 18.0 & 17.0 & 6.0 & 13.0 & 12.0 & 12.0 & 12.0 & 10.0 \\
\hline D. Quercus & 16.0 & 6.0 & 12.0 & 10.0 & 6.5 & 9.0 & 5.5 & 20.0 & 15.5 & 14.0 & 14.5 & 16.5 & 9.0 \\
\hline Ulmus & 3.0 & 1.5 & 5.5 & 5.0 & 2.5 & 1.0 & 4.0 & 5.0 & 7.5 & 3.0 & 6.5 & 1.5 & 5.5 \\
\hline Zelkova & 0.5 & 2.0 & 2.0 & 2.0 & 2.0 & 4.0 & 6.0 & 5.5 & 4.5 & 5.5 & 5.0 & 4.5 & 3.5 \\
\hline Celtys & - & - & - & - & - & - & - & - & - & - & - & - & - \\
\hline Eurya & - & - & - & - & - & - & - & - & - & - & - & - & - \\
\hline Liquidambar & 4.0 & 2.5 & 1.0 & - & 1.0 & 1.5 & 0.5 & 2.0 & 1.0 & 0.5 & 2.5 & 2.0 & 4.0 \\
\hline Sapium & - & - & - & - & - & - & - & - & - & - & - & - & 0.5 \\
\hline Rhus & 0.5 & - & - & 1.5 & - & - & 1.5 & - & - & - & 0.5 & - & - \\
\hline Acer & 1.5 & 2.0 & 0.5 & - & - & 1.5 & 1.5 & - & 0.5 & 1.0 & 1.0 & 1.5 & 0.5 \\
\hline Aesculus & 0.5 & - & - & 0.5 & - & - & - & - & - & 0.5 & 0.5 & - & - \\
\hline Hex & - & - & - & - & - & - & - & - & - & - & - & - & - \\
\hline Tilia & - & 2.0 & 1.5 & 0.5 & 1.5 & 4.5 & 1.5 & 0.5 & 1.0 & 2.0 & 1.5 & 2.5 & 1.0 \\
\hline Elaeagnus & - & - & - & - & - & - & - & - & - & - & - & - & - \\
\hline Lagerstroemia & - & - & - & - & - & - & - & - & - & - & - & - & - \\
\hline Alangium & 0.5 & - & - & - & - & - & - & - & - & - & - & - & - \\
\hline Nyssa & 0.5 & - & - & - & - & - & - & - & - & - & - & - & - \\
\hline Ericaceac & - & - & 1.0 & - & - & 0.5 & 0.5 & 1.0 & - & 1.0 & - & - & - \\
\hline Symplocos & - & - & - & - & - & - & - & 0.5 & - & - & 0.5 & - & - \\
\hline Ligustrum & - & - & - & - & - & - & - & - & - & - & - & - & - \\
\hline Weigela & - & - & - & - & - & - & - & - & - & - & - & - & - \\
\hline
\end{tabular}

Non-arboreal pollen

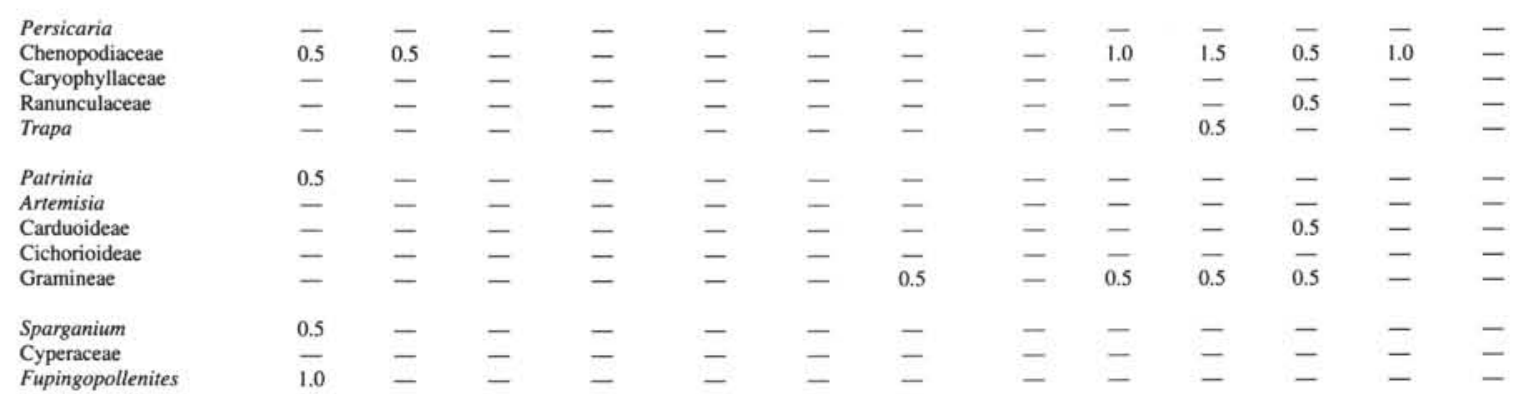

(Total $=100.0 \%$ )

Assemblage of 100 grains

\begin{tabular}{|c|c|c|c|c|c|c|c|c|c|c|c|c|c|}
\hline Abies & - & 3 & - & - & 3 & - & - & - & 1 & - & - & - & - \\
\hline Picea & 36 & 41 & 12 & 40 & 43 & 33 & 25 & 51 & 36 & 61 & 21 & 60 & 39 \\
\hline Pinus & 12 & 24 & 46 & 6 & 33 & 47 & 49 & 7 & 12 & 3 & 4 & 20 & 9 \\
\hline Tsuga & 5 & 6 & 6 & 8 & 10 & 4 & 4 & 11 & 10 & 4 & 18 & 5 & 6 \\
\hline Others & 47 & 26 & 36 & 46 & II & 16 & 22 & 31 & 41 & 32 & 57 & 15 & 46 \\
\hline $\begin{array}{l}\text { otal pollen grains } \\
\text { er gram of sediment }\end{array}$ & 3430 & 6220 & 7430 & 21.530 & 11.540 & 36,060 & 10,070 & 18,640 & 36.610 & 13,960 & 2840 & 18,080 & 26,620 \\
\hline
\end{tabular}


Table 1 (continued).

\begin{tabular}{|c|c|c|c|c|c|c|c|c|c|c|c|c|c|c|c|c|}
\hline \multicolumn{12}{|c|}{ 127-797B- } & \multicolumn{5}{|c|}{$127-797 \mathrm{C}$ - } \\
\hline $40 \mathrm{X}-2$ & $41 X-1$ & $42 \mathrm{X}-1$ & $43 \mathrm{X}-1$ & $44 X-1$ & $46 \mathrm{X}-1$ & $47 X-1$ & $48 X-2$ & $49 \mathrm{X}-1$ & $50 \mathrm{X}-1$ & $51 \mathrm{X}-1$ & $52 \mathrm{X}-1$ & $2 \mathrm{R}-1$ & $3 \mathrm{R}-1$ & $5 R-2$ & $6 \mathrm{R}-4$ & $8 \mathrm{R}-1$ \\
\hline $41-43$ & $40-42$ & $40-42$ & $41-43$ & $10-12$ & $20-22$ & $35-37$ & $39-41$ & $35-37$ & $24-26$ & $34-36$ & $34-36$ & $96-98$ & $128-130$ & 44-46 & $94-96$ & $39-4$ \\
\hline - & - & - & - & - & - & - & - & - & - & - & - & - & - & - & - & - \\
\hline- & - & - & 1.0 & - & - & - & 0.5 & 0.5 & - & - & - & - & - & - & - & - \\
\hline- & 1.0 & - & 1.5 & 1.5 & 1.5 & 0.5 & 3.5 & 3.0 & 1.5 & - & - & 0.5 & - & 5.5 & 3.5 & 3.0 \\
\hline 1.5 & 4.5 & 3.0 & - & 1.0 & 1.0 & 2.5 & 1.5 & 4.0 & 3.0 & 0.5 & - & 3.5 & 0.5 & 7.5 & 9.0 & 7.0 \\
\hline- & - & - & - & - & - & - & - & - & - & - & - & - & - & - & - & - \\
\hline - & - & - & - & - & - & - & - & - & - & - & - & - & - & 1.0 & 0.5 & - \\
\hline 1.5 & - & - & 0.5 & 1.5 & 1.0 & 0.5 & 0.5 & 1.0 & - & 1.0 & 0.5 & 2.0 & - & 2.0 & 3.0 & 1.0 \\
\hline 0.5 & 1.0 & 0.5 & 1.0 & 3.0 & - & - & - & - & - & 0.5 & - & 0.5 & - & - & - & 0.5 \\
\hline- & - & - & - & - & - & - & - & - & - & - & - & - & - & - & - & - \\
\hline- & - & - & - & - & - & - & - & - & - & - & - & - & - & - & - & - \\
\hline - & - & - & - & - & - & - & - & 0.5 & 0.5 & - & 0.5 & - & - & - & - & - \\
\hline 0.5 & - & 0.5 & 1.0 & 0.5 & - & 0.5 & - & - & 0.5 & 1.5 & - & 0.5 & - & - & 1.5 & 0.5 \\
\hline 3.0 & 7.0 & 7.5 & 7.0 & 10.5 & 2.5 & 1.5 & 1.5 & 5.5 & 8.0 & 14.0 & 15.5 & 5.5 & 7.0 & 10.0 & 5.5 & 8.0 \\
\hline 9.5 & 23.5 & 7.5 & 9.0 & 8.0 & 10.5 & 4.5 & 2.5 & 9.0 & 13.0 & 18.0 & 15.5 & 21.0 & 7.0 & 10.5 & 9.0 & 23,0 \\
\hline- & - & - & 0.5 & - & 0.5 & 0.5 & 0.5 & 1.0 & - & 0.5 & 0.5 & - & 0.5 & 0.5 & 1.0 & 2.0 \\
\hline 1.0 & 2.0 & 1.0 & 1.0 & 2.0 & 0.5 & 0.5 & - & 1.5 & 1.0 & - & 1.5 & 0.5 & - & 1.0 & 2.5 & 2.0 \\
\hline 1.5 & 5.0 & 2.0 & 1.5 & 1.5 & 1.0 & - & 0.5 & - & - & - & 0.5 & - & 1.5 & - & 0.5 & 0.5 \\
\hline 5.0 & 2.5 & 5.0 & 4.5 & 2.0 & 2.5 & 2.5 & 0.5 & 4.5 & 5.5 & 4.5 & 1.5 & 2.5 & 3.0 & 3.5 & 3.5 & 3.5 \\
\hline 0.5 & 2.0 & 1.0 & - & - & 1.0 & 0.5 & - & 0.5 & 0.5 & 0.5 & 1.0 & - & - & 1.5 & 0.5 & 1.0 \\
\hline 0.5 & 0.5 & 13.5 & 1.5 & 1.0 & 4.5 & 2.0 & 5.5 & 7.5 & 0.5 & 1.0 & 3.0 & 2.0 & 4.0 & 3.0 & 2.5 & - \\
\hline 27.0 & 15.5 & 13.5 & 29.5 & 12.5 & 10.0 & 4.0 & 3.5 & 7.5 & 12.5 & 3.5 & 5.0 & 6.0 & 3.5 & 3.0 & 8.5 & 6.5 \\
\hline 2.5 & 2.0 & 14.0 & 6.0 & 3.0 & 10.5 & 13.5 & 25.0 & 10.5 & 0.5 & 2.5 & 3.0 & 1.5 & 1.5 & 1.5 & 1.0 & 0.5 \\
\hline 17.0 & 9.0 & 12.5 & 14.5 & 22.5 & 30.0 & 37.0 & 39.0 & 24.0 & 24.0 & 21.5 & 26.5 & 27.0 & 42.0 & 31.0 & 16.0 & 8.0 \\
\hline 10.5 & 8.0 & 7.5 & 7.0 & 11.0 & 8.0 & 7.0 & 10.0 & 10.5 & 17.0 & 10.5 & 10.0 & 16.0 & 16.5 & 10.0 & 17.0 & 18.5 \\
\hline 6.0 & 4.5 & 4.5 & 3.5 & 5.5 & 6.0 & 9.5 & 3.0 & 4.0 & 5.0 & 9.0 & 5.0 & 3.5 & 3.5 & 3.5 & 6.5 & 7.0 \\
\hline 2.5 & 5.0 & 1.5 & 2.5 & 2.0 & 2.5 & 3.0 & - & 0.5 & 2.0 & 4.0 & 1.5 & 2.0 & 4.5 & 1.0 & - & 1.0 \\
\hline 0.5 & - & - & - & - & - & 0.5 & - & - & - & - & - & - & 0.5 & 0.5 & - & 1.0 \\
\hline- & - & - & - & - & - & - & - & - & - & - & - & - & - & - & - & - \\
\hline 2.0 & 2.0 & - & 4.0 & 2.0 & 2.5 & 2.0 & 0.5 & 1.5 & 2.5 & 2.5 & 2.5 & 1.5 & 4.0 & 3.0 & 7.0 & 2.0 \\
\hline- & - & - & 0.5 & - & - & - & - & - & - & - & - & - & - & - & - & - \\
\hline- & - & - & - & - & 0.5 & - & - & - & - & 0.5 & - & 1.0 & - & - & - & - \\
\hline 3.0 & 1.0 & 0.5 & - & 2.0 & 1.0 & 3.0 & 1.5 & 2.0 & 1.0 & 2.0 & 2.5 & - & 0.5 & - & 0.5 & 1.5 \\
\hline- & 0.5 & 0.5 & - & 1.0 & - & 0.5 & - & - & - & - & - & - & - & - & - & 0.5 \\
\hline- & - & - & - & - & - & - & - & - & - & 0.5 & - & - & - & 0.5 & - & - \\
\hline 1.5 & 0.5 & 1.5 & - & 1.5 & 2.0 & 1.0 & - & - & 0.5 & - & 3.0 & 2.5 & - & - & 0.5 & 1.0 \\
\hline- & - & - & 1.0 & - & - & - & - & - & - & - & - & - & - & - & - & - \\
\hline- & - & - & - & - & - & - & - & - & - & 0.5 & - & - & - & - & - & - \\
\hline- & - & - & - & - & - & - & - & - & - & - & - & - & - & - & - & - \\
\hline- & 0.5 & 0.5 & 0.5 & $=$ & $\overline{-}$ & - & $=$ & $=$ & - & - & - & - & - & - & - & - \\
\hline 1.0 & - & 0.5 & - & - & - & - & - & - & - & - & - & 0.5 & - & - & - & 0.5 \\
\hline- & - & - & - & - & - & 0.5 & - & 0.5 & - & - & - & - & - & - & - & - \\
\hline- & - & - & - & - & - & - & - & - & - & - & - & - & - & - & - & - \\
\hline- & - & - & - & - & - & - & - & - & - & - & - & - & - & - & - & - \\
\hline- & - & - & - & - & - & - & - & - & - & - & - & - & - & - & - & - \\
\hline 1.0 & 2.5 & 1.0 & 1.0 & 3.5 & - & 2.5 & 0.5 & 0.5 & 0.5 & 1.0 & - & - & - & - & 0.5 & - \\
\hline- & - & - & - & - & - & - & - & - & - & - & - & - & - & - & - & - \\
\hline- & - & - & - & - & - & - & - & - & - & - & - & - & - & - & - & - \\
\hline- & - & - & - & - & - & - & - & - & - & - & - & - & - & - & - & - \\
\hline- & - & - & - & - & - & - & - & - & - & - & - & - & - & - & - & - \\
\hline- & - & - & - & - & - & - & - & - & - & - & - & - & - & - & - & - \\
\hline- & - & - & - & 0.5 & - & - & - & - & - & - & - & - & - & - & - & - \\
\hline- & - & - & - & - & - & - & - & - & - & - & - & - & - & - & - & - \\
\hline- & - & 0.5 & - & - & 0.5 & - & - & - & 0.5 & - & - & - & - & - & - & - \\
\hline- & - & - & - & - & - & - & - & - & - & - & - & - & - & - & - & - \\
\hline- & - & - & - & - & - & - & - & - & - & - & 0.5 & - & - & - & - & - \\
\hline 0.5 & - & - & - & 0.5 & - & - & - & - & - & - & 0.5 & - & - & - & - & - \\
\hline- & - & - & - & - & - & - & - & - & - & - & - & - & - & - & - & 1 \\
\hline 43 & 42 & 42 & 45 & $\overline{59}$ & 32 & $\overline{17}$ & $\overline{14}$ & $\overline{36}$ & 47 & 58 & 36 & $\overline{35}$ & 20 & 31 & 15 & 31 \\
\hline 22 & 10 & 11 & 18 & 11 & 23 & 41 & 22 & 31 & 21 & 15 & 29 & 27 & 45 & 42 & 61 & 34 \\
\hline 7 & 7 & - & 4 & 4 & 3 & 3 & 2 & 10 & 8 & 10 & 4 & 6 & - & 9 & 5 & 7 \\
\hline 28 & 41 & 47 & 33 & 26 & 42 & 39 & 62 & 23 & 24 & 17 & 31 & 32 & 35 & 18 & 19 & 27 \\
\hline 15.220 & 9680 & 20,990 & 13.860 & 8800 & 26.400 & 13,160 & 38.150 & 13,200 & 33,230 & 25,060 & 31,550 & 31.500 & 13,210 & 16,070 & 17.770 & 14,990 \\
\hline
\end{tabular}


Table 1 (continued).

\begin{tabular}{|c|c|c|c|c|c|c|c|c|}
\hline \multirow{3}{*}{$\begin{array}{c}\text { Hole: } \\
\text { Core, section: } \\
\text { Interval }(\mathrm{cm}) \text { : }\end{array}$} & \multicolumn{8}{|c|}{$127-797 C-$} \\
\hline & $11 R-1$ & $18 \mathrm{R}-1$ & $19 \mathrm{R}-5$ & $20 \mathrm{R}-1$ & $22 \mathrm{R}-2$ & $25 R-1$ & $31 \mathrm{R}-4$ & $33 R-1$ \\
\hline & $9-11$ & $64-66$ & $67-69$ & $92-94$ & $119-121$ & $131-133$ & $101-103$ & $103-105$ \\
\hline \multicolumn{9}{|l|}{ Arboreal pollen } \\
\hline Larix & - & - & - & - & - & - & - & - \\
\hline Dacrydium & - & - & - & - & - & - & - & - \\
\hline Metasequoia & 1.0 & 1.5 & 2.0 & - & - & - & 1.0 & 3.0 \\
\hline Taxodiaceae & 1.5 & 0.5 & 2.0 & - & - & - & - & - \\
\hline Sciadopitys & - & - & - & - & - & - & - & - \\
\hline Cunninghamia & - & - & - & - & - & - & - & - \\
\hline Cupressaceae & - & 2.0 & - & - & - & - & - & - \\
\hline Ephedra & - & - & 0.5 & - & - & - & - & - \\
\hline Ginkgo & - & - & - & - & - & - & - & - \\
\hline Sarix & - & - & - & - & - & - & - & - \\
\hline Myrica & - & - & - & - & - & - & - & - \\
\hline Juglans & 4.5 & 2.5 & 3.0 & 4.0 & 0.5 & 3.0 & 1.0 & 2.0 \\
\hline Pterocarya & 6.5 & 12.5 & 11.0 & 7.0 & 11.5 & 11.0 & 12.0 & 7.0 \\
\hline Carya & 13.5 & 23.5 & 16.0 & 16.5 & 21.5 & 20.5 & 20.0 & 16.0 \\
\hline Engelhardia & 1.0 & 2.0 & 1.5 & 1.5 & 1.0 & 2.0 & 1.0 & 2.0 \\
\hline Alnus & 1.0 & 4.5 & 3.0 & 3.0 & 3.5 & 6.0 & 1.0 & 2.0 \\
\hline Betula & - & 0.5 & - & 2.5 & 0.5 & - & 1.0 & - \\
\hline Carpinus & 6.5 & 2.0 & 8.0 & 4.0 & 2.5 & 3.0 & 2.0 & 8.0 \\
\hline Corylus & 0.5 & - & 0.5 & - & 0.5 & - & - & - \\
\hline Castanea & 1.0 & - & - & - & - & - & - & - \\
\hline Fagus & 17.0 & 17.5 & 23.5 & 29.0 & 19.0 & 19.5 & 30.0 & 37.0 \\
\hline Pasania & - & - & - & - & - & - & - & - \\
\hline E. Quercus & 6.5 & 3.0 & - & 4.0 & 8.0 & - & - & - \\
\hline D. Quercus & 16.5 & 5.0 & 7.0 & 4.0 & 10.5 & 12.0 & 18.0 & 3.0 \\
\hline Ulmus & 11.0 & 13.5 & 12.0 & 11.5 & 11.5 & 17.0 & 7.0 & 10.0 \\
\hline Zelkova & 2.5 & - & 0.5 & 4.0 & 3.5 & 1.0 & 1.0 & - \\
\hline Celtys & - & - & - & - & - & - & - & - \\
\hline Eurya & - & - & - & - & - & - & - & - \\
\hline Liquidambar & 3.5 & 4.0 & 2.0 & 6.0 & 2.5 & 2.0 & 1.0 & 1.0 \\
\hline Sapium & - & - & - & - & - & - & - & - \\
\hline Rhus & 0.5 & 1.0 & - & - & 0.5 & - & - & 1.0 \\
\hline Acer & - & 1.0 & 5.0 & 1.0 & - & 1.0 & - & 2.0 \\
\hline Aesculus & - & - & - & - & - & 1.0 & - & - \\
\hline Hex & - & - & - & - & - & - & - & - \\
\hline Tilia & 2.0 & 3.5 & 1.5 & 2.0 & 2.5 & 1.0 & 4.0 & 6.0 \\
\hline Elaeagnus & - & - & - & - & - & - & - & - \\
\hline Lagerstroemia & - & - & - & - & - & - & - & - \\
\hline Alangium & - & - & - & - & - & - & - & - \\
\hline Nyssa & - & - & - & - & - & - & - & - \\
\hline Ericaceae & 0.5 & - & - & - & - & - & - & - \\
\hline Symplocos & - & - & - & - & - & - & - & - \\
\hline Ligustrum & - & - & - & - & 0.5 & - & - & - \\
\hline Weigela & - & - & 0.5 & - & - & - & - & - \\
\hline \multicolumn{9}{|l|}{ Non-arboreal pollen } \\
\hline Persicaria & - & - & - & - & - & - & - & - \\
\hline Chenopodiaceae & - & - & - & - & - & - & - & - \\
\hline Caryophyllaceae & - & - & - & - & - & - & - & - \\
\hline Ranunculaceae & - & - & - & - & - & - & - & - \\
\hline Trapa & - & - & - & - & - & - & - & - \\
\hline Patrinia & - & - & - & - & - & - & - & - \\
\hline Artemisia & - & - & - & - & - & - & - & - \\
\hline Carduoideae & - & - & - & - & - & - & - & - \\
\hline Cichorioideae & - & - & - & - & - & - & - & - \\
\hline Gramineae & - & - & 0.5 & - & - & - & - & - \\
\hline Sparganium & 0.5 & - & - & - & - & - & - & - \\
\hline Cyperaceae & - & - & - & - & - & - & - & - \\
\hline Fupingopollenites & 2.5 & - & - & - & - & - & - & - \\
\hline
\end{tabular}

$($ Total $=100.0 \%)$

Assemblage of 100 grains

\begin{tabular}{lcccccccc} 
Abies & $\overline{29}$ & $\overline{46}$ & $\overline{60}$ & 32 & 23 & 52 & 54 & 48 \\
$\begin{array}{l}\text { Picea } \\
\text { Pinus }\end{array}$ & 36 & 14 & 14 & 6 & 8 & 11 & 15 & 11 \\
Tsuga & 4 & 5 & 10 & 8 & 6 & 1 & 12 & 15 \\
$\begin{array}{l}\text { Others } \\
\text { Total pollen grains }\end{array}$ & 31 & 35 & 16 & 54 & 63 & 36 & 19 & 26 \\
per gram of sediment & 8370 & 3550 & 2200 & 2400 & 4210 & 3390 & 550 & 440 \\
\hline
\end{tabular}




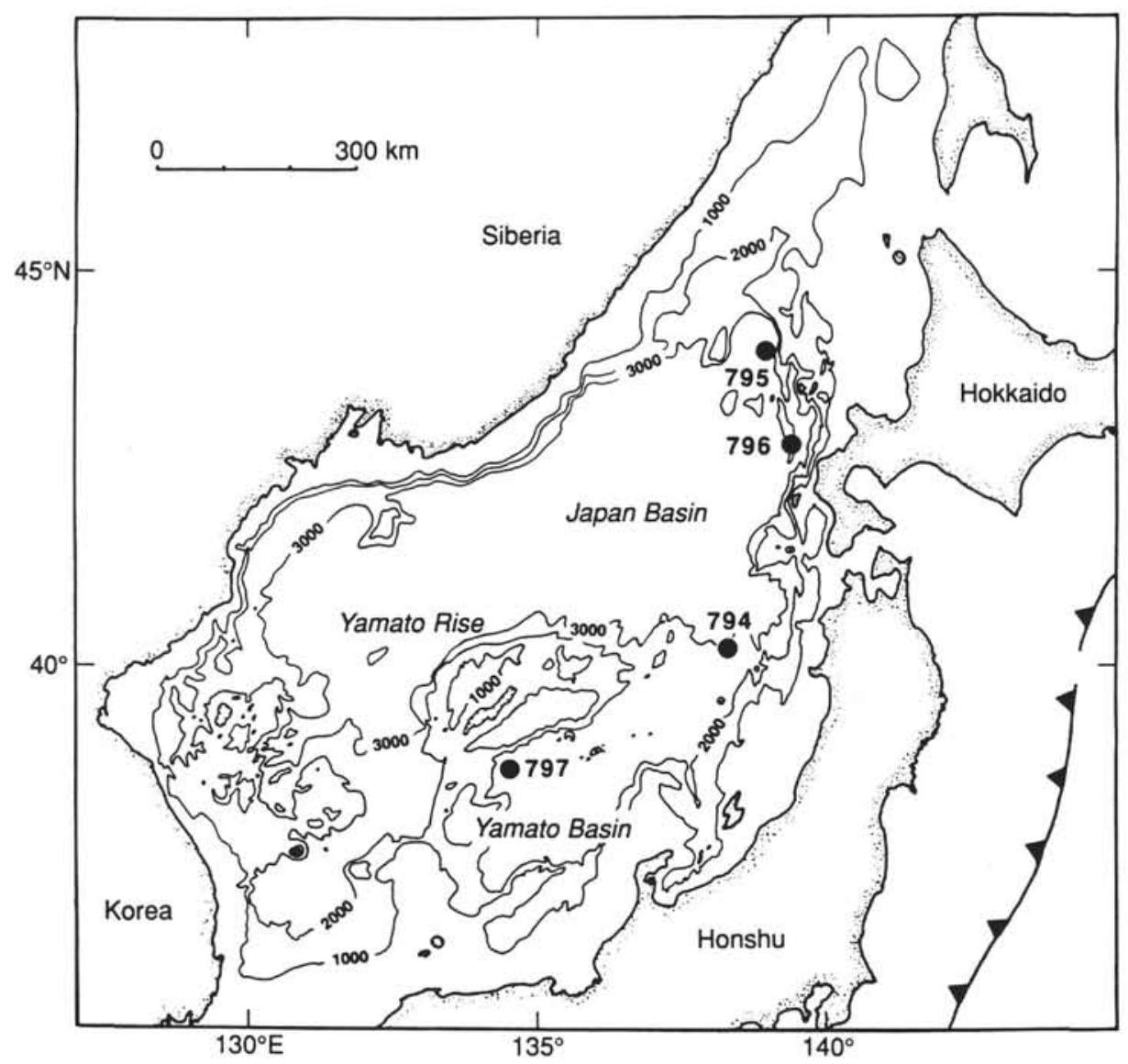

Figure 1. Map of the Japan Sea showing the locations of Leg 127 Sites 794-797.

\section{Site 797}

This site is located in the south-central Japan Sea within the Yamato Basin, about $300 \mathrm{~km}$ from the western coast of Honshu (Fig. 1). Drilling reached acoustic basement through thick marine sequence at 554 meters below seafloor (mbsf). The lower sediments in this sequence are composed of interlayered basaltic and sedimentary rocks. Drilling $350 \mathrm{~m}$ into this interlayered rock recovered not only igneous rocks but also some horizons of sedimentary rocks. Pollen fossils obtained from these lower, older sediments are different from those at the other three sites of Leg 127.

The results of pollen analyses of Site 797 are summarized in Figure 5, which shows the major floral components. Pterocarya, Carya, and D. Quercus are generally abundant at Site 797. However, Carya shows a rather high value from Section 127-797C-8R-1 downhole. Fagus occurs in every sample and especially shows a very high value above Section 127-797B-43X-1 and below Section 127-797C11R-1. E. Quercus occurs abundantly at upper horizons and becomes especially high in abundance from Section 127-797B-44X-1 to Core 127-797C-5R. As can be seen in Figure 5, Pasania is abundant locally in upper horizons, with its highest value of $25 \%$ occurring in Sample 127-797B-48X-2, 39-41 cm.

The preservation of pollen grains is very good until above Section 127-797C-20R-1, but takes a sudden change for the worse from Section 127-797C-22R-1 to the lower end of this hole. Therefore, sufficient pollen grains for statistical treatment were not obtained in many samples. However, there are some samples in which fungi spores were very abundant. The analyses of these horizons are summarized in Table 2. In addition, a dinoflagellate cyst was found in Sample 127-797C-30R-2, 73-75 cm.

\section{POLLEN FLORA OF MIOCENE SEDIMENTS}

\section{An Outline of Northeast Japan}

Many Neogene sequences from the Japan Sea side of Honshu have been analyzed for palynological studies (Yamanoi, 1976, 1978a, 1978b, 1979, 1983, 1984; Yamanoi and Tsuda, 1986). These studies have revealed several principal trends on palynofloral changes during the Neogene. Moreover, five pollen zones are also recognized in these Neogene sequences on the basis of floral change. The five pollen zones are termed the NP1 to NP5 zones in upward sequence. Zones NP1 to NP4 are assigned a Miocene age, except for the upper part of Zone NP4, which is considered to be nearing Pliocene in age (Yamanoi, 1989). The characteristics of Miocene pollen zones recognized in northeast Japan are summarized below.

Zone NP1 consists mainly of deciduous broad-leaved trees and coniferous trees such as Juglans, Pterocarya, Betula, Carpinus, Fagus, D. Quercus, Picea, and Pinus (Yamanoi, 1989). Moreover, Carya is one of the most important elements of this zone (Yamanoi, in press). Therefore, the paleotemperature of this zone is estimated as temperate or rather cool-temperate. The lowermost limit of this zone is still unknown.

Zone NP2 is characterized by the abundance of Carya, E. Quercus, and Liquidambar, and also by some presence of Dacrydium (Yamanoi, 1989). Fossil pollen of mangrove plants are included in this zone in Southwest Japan (Yamanoi et al., 1980; Yamanoi, 1984; Yamano and Tsuda, 1986) and the mean winter temperature for these regions during the early middle Miocene is estimated to be nearly $16^{\circ} \mathrm{C}$ higher than today (Yamanoi, 1989). Such a warm event can be considered as a "tropical spike," which is named for the paleotemperature rising like 


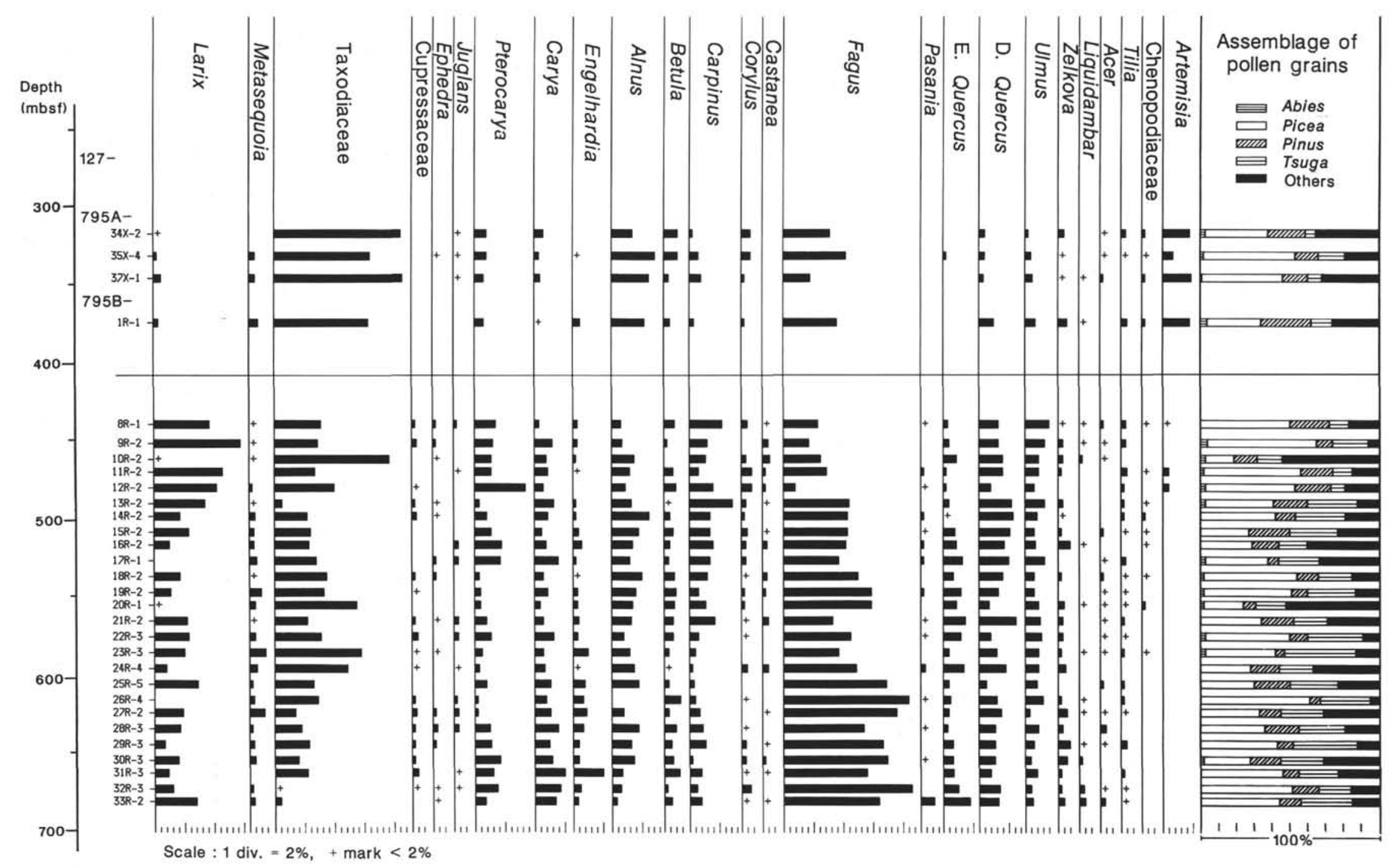

Figure 2. Pollen diagram showing major floral components of Site 795. Two fixed sums are represented: all pollen except for the vesiculate Pinaceae, $N=200$; and all pollen for the "Pinaceae vs, other" diagram, $N=100$ (shown on the right). 


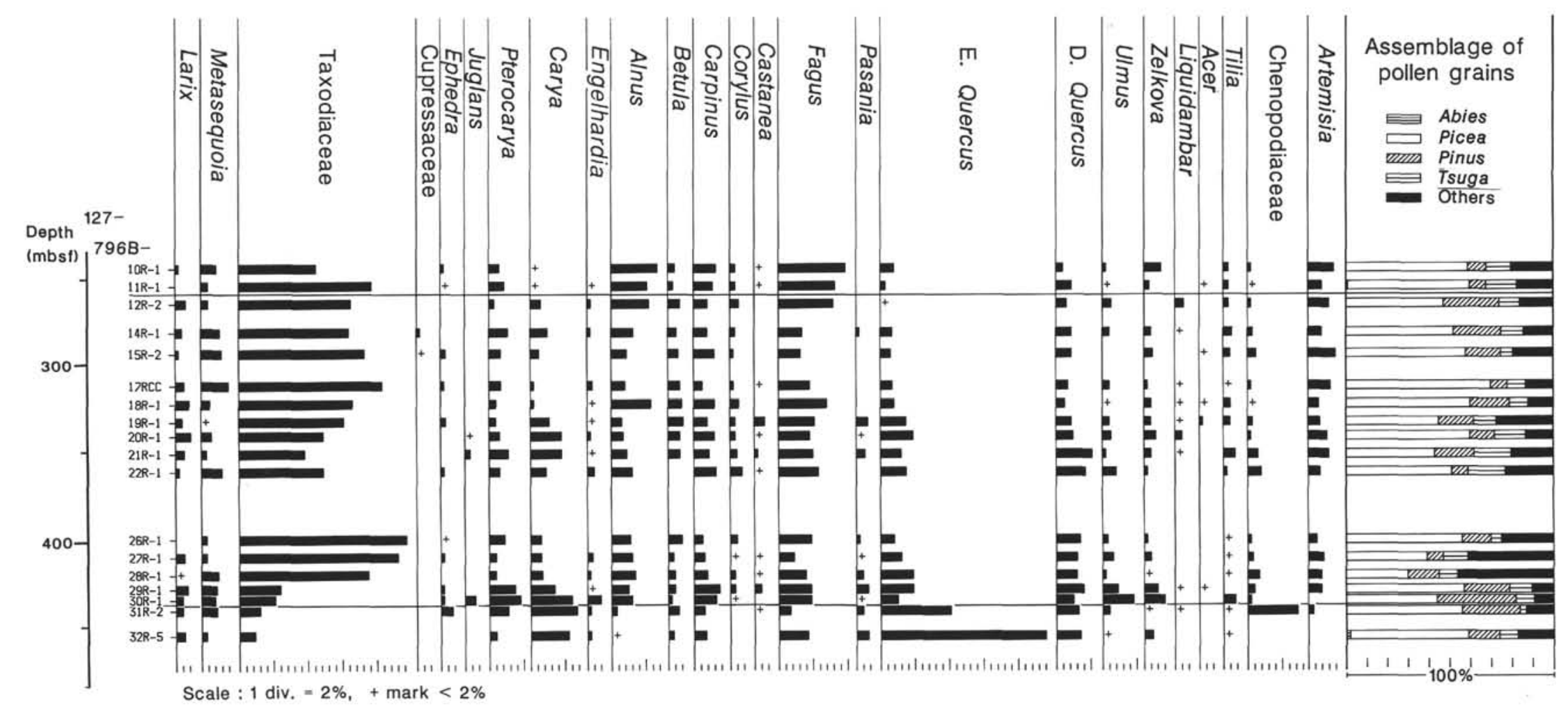

Figure 3. Pollen diagram showing major floral components of Site 796. See Figure 2 for further explanation. 


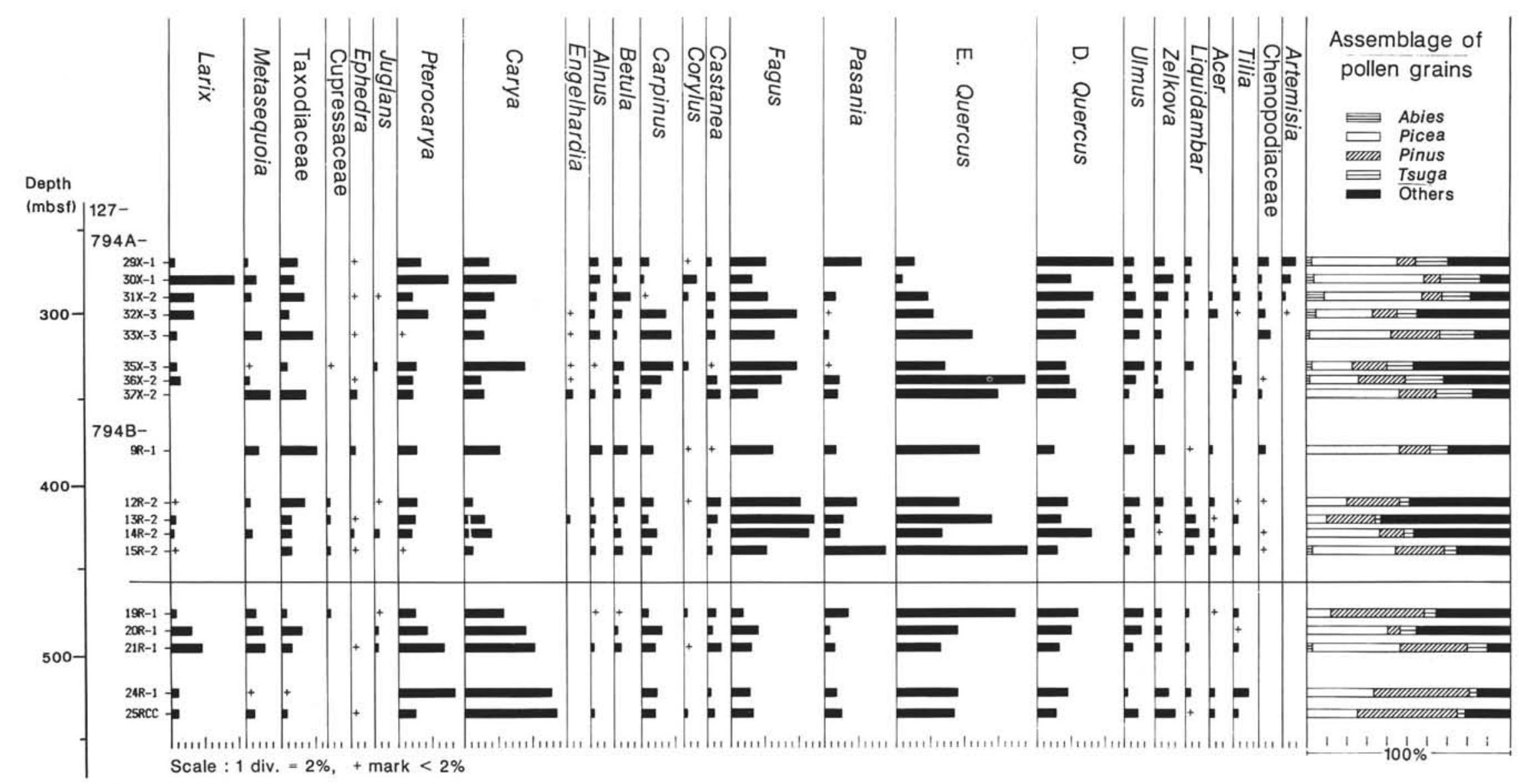

Figure 4. Pollen diagram showing major floral components of Site 794. See Figure 2 for further explanation. 


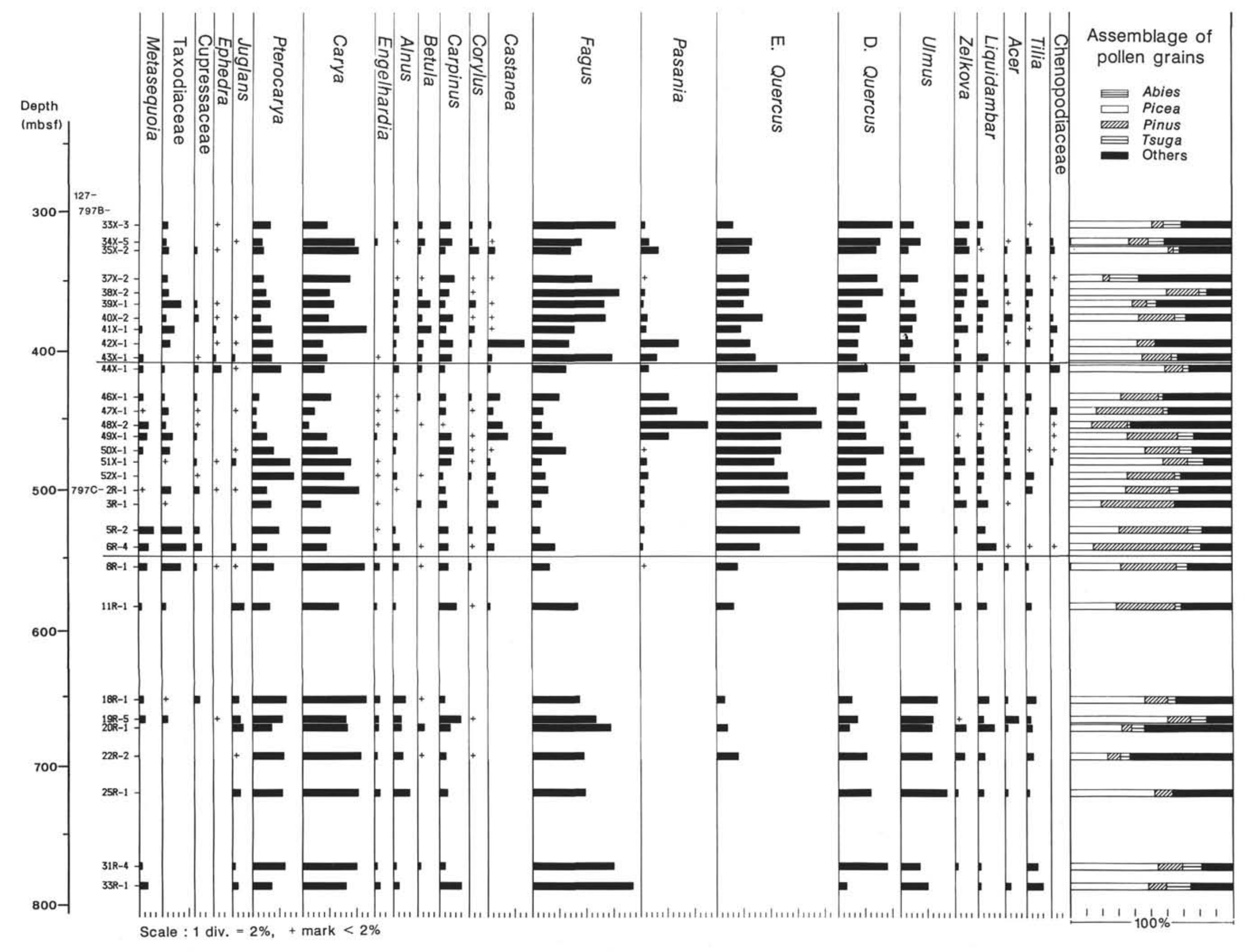




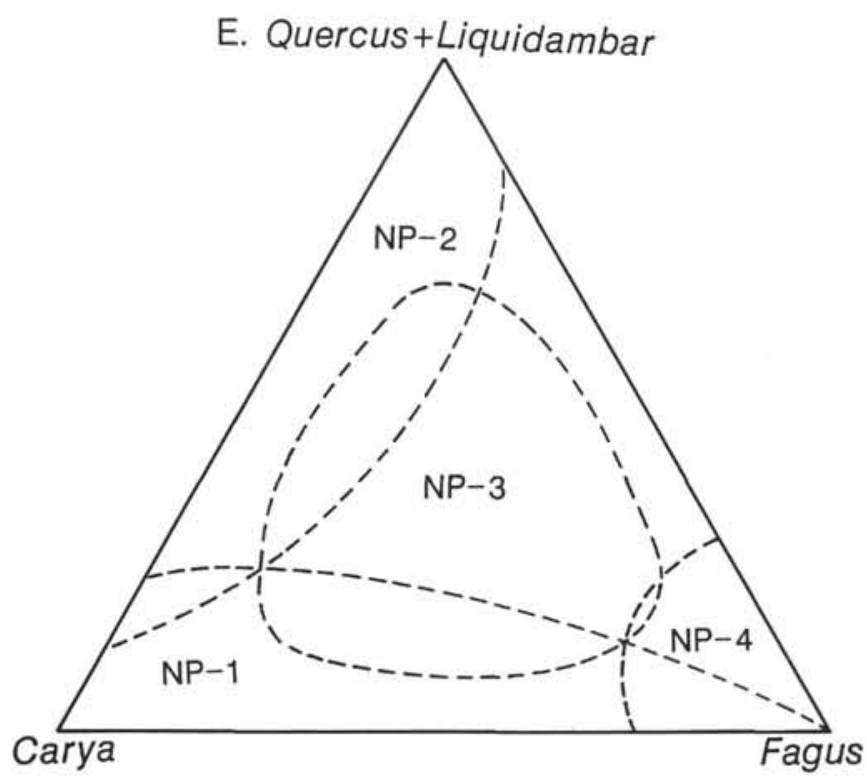

Figure 6. Ternary diagram showing the clusters of the components of the essential pollen members for Miocene pollen zones in northeast Japan.

a quick pulse (Itoigawa, 1989). Therefore, Zone NP2 contains the most abundant warm floral elements among all five pollen zones.

Zone NP3 is characterized by a high proportion of Carya and Liquidambar. The occurrence of E. Quercus becomes low, and in contrast, Fagus and Taxodiaceae are higher in percentages (Yamanoi, 1989). The paleotemperature indicated by this palynoflora is cooler than that of Zone NP2, but warmer than it is at present.

Zone NP4 is characterized by the abundance of Taxodiaceae and Fagus. The lower limit of this zone is defined by a very sharp decrease in Carya and Liquidambar (Yamanoi, 1978a). This boundary is clearly recognized in many localities of northeast Japan. Therefore, the writer named this horizon the "Funakawa floral change plane" (Yamanoi, 1978a).

As described above, there are several essential members that caused the palynofloral change in northeast Japan during the Miocene. Major floral changes are thus recognized by relative variations of such essential genera as Carya, Fagus, E. Quercus, and Liquidambar: These four pollen genera are divided first into three components, Liquidambar plus E. Quercus (warm element), Carya (moderate element), and Fagus (cool element) so that each pollen zone can be recognized through cluster plots on a ternary diagram such as the one shown in Figure 6.

\section{Miocene Pollen Flora of Leg 127}

\section{Site 795}

The pollen flora of this site is divided into two local pollen zones (Fig. 2). The boundary of these zones is expected to occur at any horizon between Sections 127-795B-1R and 127-795B-8R-1. However, this boundary cannot be determined with accuracy because of low recovery for this interval. The upper local pollen zone is characterized by an abundance of Taxodiaceae, Alnus, and Artemisia. In the lower local pollen zone, Larix, Carya, Carpinus, E. Quercus, and Ulmus are more dominant than in the upper local pollen zone.

The components of the essential member were plotted on a ternary diagram (Fig. 7). As shown in Figure 7, the upper and lower local pollen zones can be correlated with Zones NP4 and NP3 of northeast Japan, respectively. The points belonging to each zone do not separate sharply at their boundaries. The reason is considered to be that Fagus occurred dominantly in both zones, as this site is located farther north from northeast Japan.

\section{E. Quercus+Liquidambar}

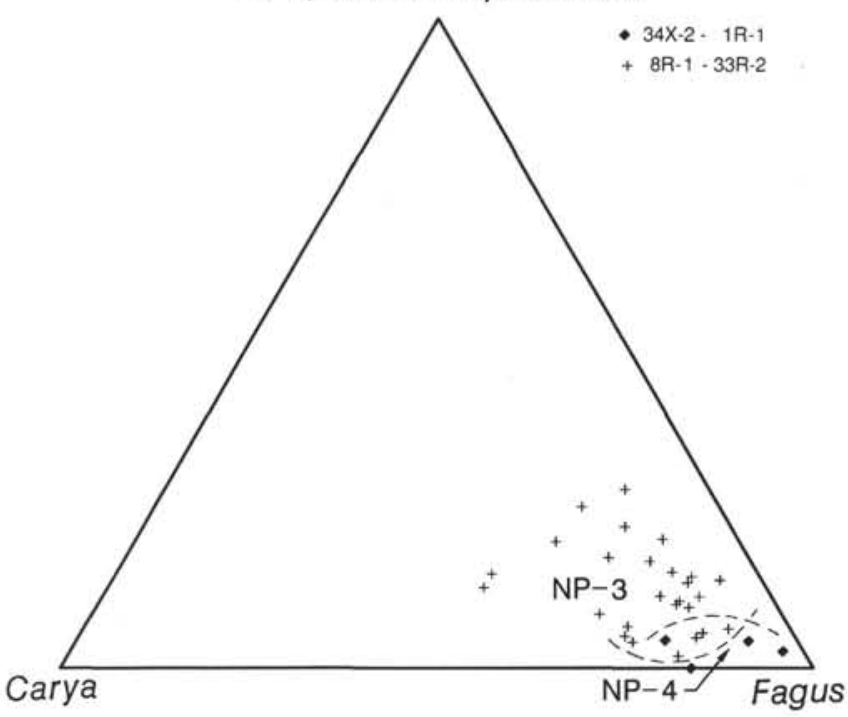

Figure 7. Ternary diagram showing the three-composition ratio of four essential pollen members for Site 795. Closely clustered plots regarded as representing entities of pollen floras, and their correspondence with the pollen zones of northeast Japan, are shown in Figure 6.

\section{Site 796}

A remarkable floral change can be seen in the older sediments at this site (Fig. 3). E. Quercus shows a high value and Carya is fairly abundant in Section 127-796B-31R-2 and its lower horizons. Therefore, this floral boundary is estimated to occur between Sections 127-796B-30R-1 and 127-796B-31R-2 (Fig. 3). The pollen floras marking this boundary are considered to correspond with Zones NP3 and NP2 of northeast Japan.

The components of the essential member were plotted on a ternary diagram (Fig. 8). From this we can see that there is a wide difference in the components between the horizons above Section 127-796B30R-1 and below Section 127-796B-31R-2. Moreover, Figure 8

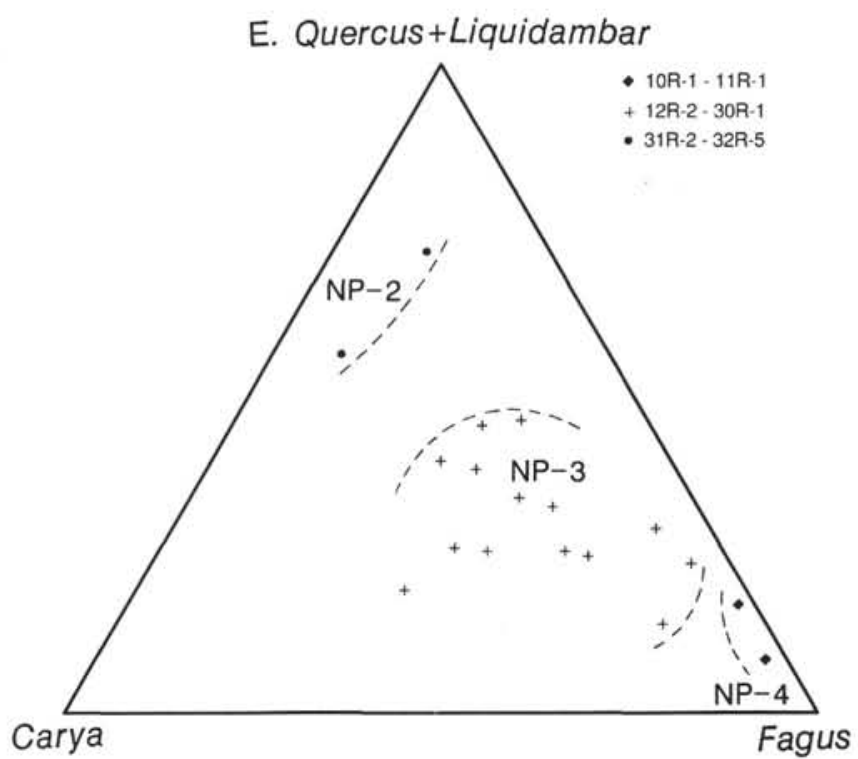

Figure 8. Ternary diagram showing the three-composition ratio of four essential pollen members for Site 796. See Figure 7 for explanation. 
Table 2. Occurrences, color, and preservation of pollen grains and abundance of fungi spores in the lowermost cores of Hole 797C.

\begin{tabular}{|c|c|c|c|c|}
\hline $\begin{array}{l}\text { Core, section, } \\
\text { interval }(\mathrm{cm})\end{array}$ & $\begin{array}{l}\text { Total pollen grains } \\
\text { per gram of sediment }\end{array}$ & $\begin{array}{c}\text { Color of } \\
\text { pollen grain }\end{array}$ & $\begin{array}{l}\text { Preservation of } \\
\text { pollen grain }\end{array}$ & $\begin{array}{l}\text { Abundance of } \\
\text { fungi spores }\end{array}$ \\
\hline \multicolumn{5}{|l|}{$127-797 \mathrm{C}$ - } \\
\hline $18 R-1,64-66$ & 3550 & Light brown & Good & Few \\
\hline $19 \mathrm{R}-5,67-69$ & 2200 & Light brown & Rare & \\
\hline $20 \mathrm{R}-1,92-94$ & 2400 & Brown & Good & Few \\
\hline $22 \mathrm{R}-1,53-55$ & 10 & Black & Poor & Rare \\
\hline $22 \mathrm{R}-2,119-121$ & 4210 & Light brown & Moderate to poor & Few \\
\hline $22 \mathrm{R}-\mathrm{CC}, 12-14$ & 30 & Black & Poor & Few \\
\hline $23 \mathrm{R}-3,35-37$ & 120 & Dark brown & Moderate to poor & Few \\
\hline $23 \mathrm{R}-5,83-85$ & 10 & Black & Very poor & Common \\
\hline $25 \mathrm{R}-1,13 \mathrm{I}-133$ & 3390 & Dark brown & Moderate to poor & Abundant \\
\hline $25 \mathrm{R}-6,46-48$ & 90 & Dark brown & Poor & Abundant \\
\hline $30 \mathrm{R}-2,73-75$ & 130 & Dark brown & Moderate to poor & Common \\
\hline $31 \mathrm{R}-4,101-103$ & 550 & Brown & Moderete to poor & Few \\
\hline $33 \mathrm{R}-1,103-105$ & 440 & Brown & Poor & Few \\
\hline $34 \mathrm{R}-3,89-91$ & 20 & Brown & Moderate to poor & Few \\
\hline $34 \mathrm{R}-6,104-106$ & 0 & - & - & - \\
\hline $37 \mathrm{R}-1,38-40$ & 340 & Dark brown & Very poor & Few \\
\hline $37 \mathrm{R}-3,148-150$ & 30 & Dark brown & Poor & Few \\
\hline $37 R-4,61-63$ & 210 & Dark brown & Very poor & Few \\
\hline $37 \mathrm{R}-6,140-142$ & 190 & Dark brown & Very poor & Rare \\
\hline $41 \mathrm{R}-1,145-147$ & 30 & Black & Very poor & Common \\
\hline $4 \mid \mathrm{R}-3,39-41$ & 30 & Black & Very poor & Common \\
\hline
\end{tabular}

shows that the components of Sections 127-796B-10R-1 and 127796B-11R-1 belong to Zone NP4, as compared with the floral component of northeast Japan (Fig. 6).

Thus, the boundary between Zones NP3 and NP4 of this site can be drawn between Sections 127-796B-11R-1 and 127-796B-12R-2 (Fig. 3). The age of the NP4/NP3 boundary is estimated to be about $7 \mathrm{Ma}$ on the basis of marine microfossil data for this site (Tamaki, Pisciotto, Allan, et al., 1990).

\section{Site 794}

The pollen flora of this site can be divided into the upper and lower local pollen zones (Fig. 4). The boundary must be between Sections 127-794B-15R-2 and 127-794B-1R-2. However, the boundary cannot be determined in detail because the samples of this horizon are not yet available. Fagus and E. Quercus are dominant in the upper local pollen zone (Fig. 4). In contrast, Pterocarya, Carya, and E. Quercus occur dominantly in the lower local pollen zone.

The essential member of the pollen flora of Site 794 was plotted for their components on a ternary diagram (Fig. 9). These components are classified into two clusters on the diagram. One cluster remarks the lower local pollen zone (Sections 127-794B-19R-1 to 127-794B-25R$\mathrm{CC}$ ) is correlated to the pollen flora of Zone NP2 of northeast Japan. Since the lower local pollen zone is correlated to Zone NP2, the upper local pollen zone (Sections 127-794A-29X-1 to 127-794B-15R-2) must correspond with Zone NP3 of northeast Japan. However, as can be seen in Figure 9, many points of the components for the upper local pollen zone shift upward on the diagram, considering that they belong to Zone NP3. This shift is caused by a high value of E. Quercus in this upper zone. Such a phenomenon probably reflects the sedimentational character of E. Quercus pollen grains in an offshore region at this time.

The age of the NP3/NP2 boundary is inaccurate as yet, but is estimated to be between 12.3 Ma and 14.5 Ma on the basis of marine microfossil data at Site 794 (Tamaki, Pisciotto, Allan, et al., 1990). In addition, the NP4/NP3 boundary is expected to come above the uppermost core shown in Figure 4.

\section{Site 797}

The pollen flora of this site is divided into the upper, middle, and lower local pollen zones. The boundaries of the three local pollen

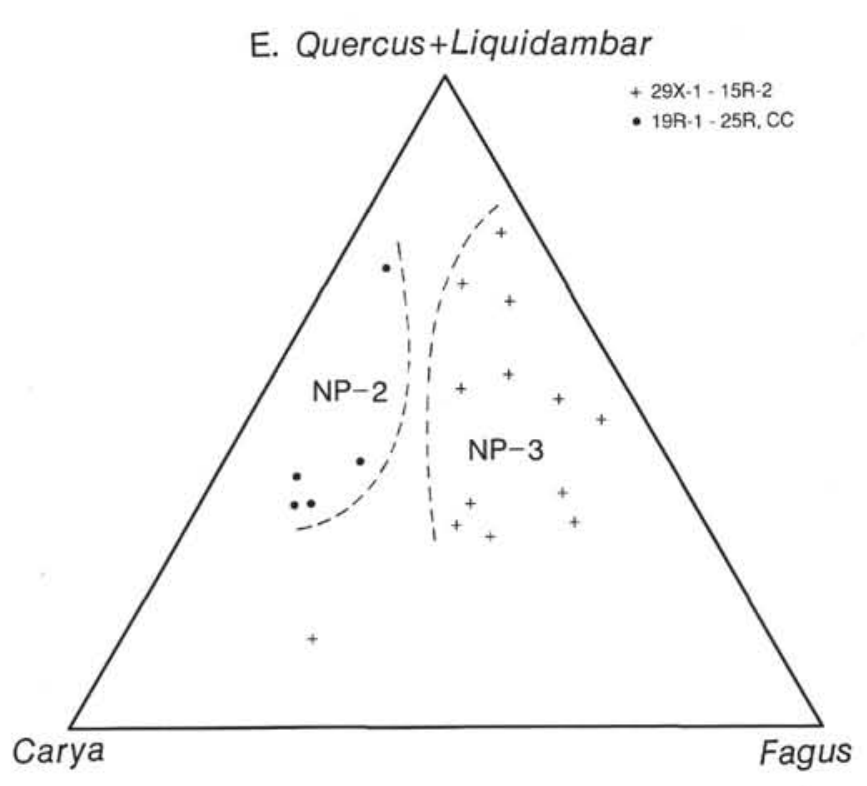

Figure 9. Ternary diagram showing the three-composition ratio of four essential pollen members for Site 794. See Figure 7 for explanation.

zones occur between Sections 127-797B-43X-1 and 127-797B-44X1, and between Sections 127-797C-6R-4 and 127-797C-8R-1 (Fig. 5). In the upper local pollen zone, Carya and Fagus occur abundantly, with fairly abundant occurrences of E. Quercus and D. Quercus. The middle local pollen zone is characterized by the dominance of $\mathrm{E}$. Quercus and the lower abundance of Fagus. Pollen of Pterocarya, Carya, and Pasania are abundant locally in this zone.

The components of the essential member were plotted on a ternary diagram (Fig. 10). The components are classified clearly into three clusters. These clusters, which are in accordance with the three local pollen zones, can be correlated with Zones NP1, NP2, and NP3 of northeast Japan (Fig. 10).

The age of the NP3/NP2 boundary of this site is about $13 \mathrm{Ma}$, based on associated marine microfossils (Tamaki, Pisciotto, Allan, et al., 
1990). As discussed previously, the age of the boundary of the NP4/NP3 is estimated to be about $7 \mathrm{Ma}$ at Site 796. Thus, the flora must be changed to the flora of Zone NP4 in the upper part of the upper local pollen zone. The components of the essential member occurring in Section 127-797B-33X-3 (Fig. 10) may belong to Zone NP4, but this is a subject for future study. The age of the NP2/NP1 boundary, which is not determined with accuracy because of the lack of other microfossil data (Tamaki, Pisciotto, Allan, et al., 1990), is assumed to fall between $17 \mathrm{Ma}$ and $18.5 \mathrm{Ma}$. This is the first time a complete range of the pollen flora of early middle Miocene Zone NP2 has been obtained.

The horizon containing an abundance of E. Quercus and Pasania in Zone NP2 at Site 797 may be closely related to the early middle Miocene "tropical spike" (Itoigawa, 1989) at about $16 \mathrm{Ma}$.

In Zone NP1 of this site, the flora is characterized by the abundance of Carya, Fagus, and Ulmus. This is thought to reflect a cool or cool-temperate paleotemperature. Pollen grains are not only poor preserved but also occur in lower numbers in the lower part of Zone NP2 (Table 2). However, many kinds of fungal spores occur abundantly in these horizons (Sections 127-797C-25R-1 and 127-797C25R-6). This fact indicates that much terrestrial matter was deposited at this site at that time, because many fungi live in well-ventilated, dried organic soil.

\section{Pollen Stratigraphy}

The pollen zones recognized at each site can be correlated as illustrated in Figure 11. Four pollen zones are recognized as a different type of flora. The ages obtained for the boundaries of these zones are about $7 \mathrm{Ma}, 13 \mathrm{Ma}$, and 17-18.5 Ma (Fig. 11). Although the existence of Zone NP1 was confirmed at Site 797, this zone was not recognized in other Leg 127 sites because drilling did not penetrate sediments of the appropriate age. At Site 795, drilling did not reach sufficient depth even for Zone NP2.

Zone NP4 is correlated with the Mitoku-type flora (Tanai, 1961) and Zone NP2 can be correlated with the Daijima-type flora (Tanai, 1961). However, Zone NP3 may correspond to a barren zone of mega-plant fossils. The Aniai-type flora has been placed below the position of the Daijima-type flora (Tanai, 1961). Suzuki (1989) has

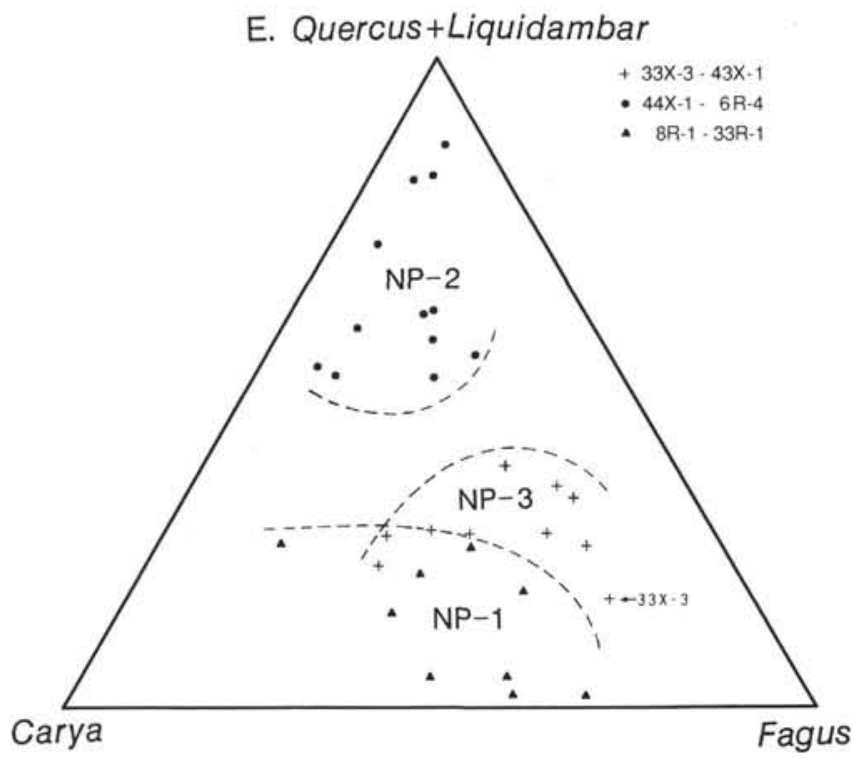

Figure 10. Ternary diagram showing the three-composition ratio of four essential pollen members for Site 797. See Figure 7 for explanation. proposed the existence of the Aniai-Daijima mixed-type flora between the Daijima-type and Aniai-type floras. Moreover, the ages of the Daijimai-type and Aniai-type floras have been assigned to earliest Miocene to early middle Miocene (ca. 13-22 Ma) and latest Oligocene to earliest Miocene (ca..22-26 Ma), respectively, by Kano and Yanagisawa (1989). However, as to the Aniai-type flora, the relations among the floral component, stratigraphic positions, and time range have not been clearly resolved. Therefore, the pollen flora of Zone NP1 may be useful in solving the problems associated with recognition of the "Aniai-type flora."

Certain differences are exhibited among the floral components of the pollen zones correlated in Figure 11. Concerning Zone NP3, Fagus and Carya have commonly abundant occurrences at individual sites. However, Taxodiaceae and Larix occur dominantly at Site 795, Taxodiaceae occurs very abundantly at Site 796, and E. Quercus is fairly abundant in Site 797. These floral differentiations of each site reflects the sedimentational character of pollen grains and the vegetational habitats on land areas of that time. The pollen data of Zone NP3 were obtained in this study, and those that have been obtained on land areas (Yamanoi, 1976, 1978a, 1978b, 1989) have been assembled. These data show the distribution of pollen that would be expected to be occur abundantly in Zone NP3 (Fig. 12). Figure 13 illustrates Zone NP2 in much the same way as Figure 12 does for Zone NP3. These maps will become more precise with increasing pollen data from the Japan Sea area.

\section{SUMMARY}

Approximately 100 samples of Miocene sediments were analyzed palynologically at four Leg 127 sites in the Japan Sea (Fig. 1). The pollen components and total pollen grains per gram of dry sediment of each sample are summarized in Table 1. The floral components of major pollen taxa of each site are illustrated in Figures 2 through 5.

Neogene pollen floras, which have been established as a standard pollen zones in northeast Japan, are briefly described. These standard pollen zones are represented by a cluster composition for essential members by using a ternary diagram (Fig. 6). The essential member of the local pollen floras of each site was plotted for components on ternary diagrams (Figs. 7-10). Closely clustered plots on these diagrams representing local floras at ODP sites can be correlated with the standard pollen zones of northeast Japan (Fig. 6).

The pollen zones of each ODP site are correlated as shown in Figure 11. The age of the boundaries of the pollen zones are about 7 $\mathrm{Ma}, 13 \mathrm{Ma}$, and 17-18.5 Ma (Fig. 11).

The pollen data of Zones NP3 and NP2, which were obtained in this study, and data obtained from land areas, were combined. These data are summarized in two maps showing distribution of the pollen which are expected to occur abundantly (Figs. 12 and 13).

\section{ACKNOWLEDGMENTS}

I wish to thank the Ocean Drilling Program and Kensaku Tamaki, co-chief scientist on Leg 127, for giving me the opportunity to conduct this study. I also thank Alfred Traverse and anonymous reviewers for providing valuable criticism and helpful advice.

\section{REFERENCES}

Itoigawa, J., 1989. Tropical spike in early Middle Miocene (ca. 16 Ma) of Southwest Japan. Int. Symp. Pacific Neogene Continental and Marine Events. Nanjing Univ. Press, IGCP-246: 19-26.

Kano, K., and Yanagisawa, Y., 1989. Ages of the Aniai-type and Daijima-type floras in Japan. Chishitsu Chosasho Geppo, 40:647-653.

Suzuki, K., 1989. On the plant biostratigraphy of the Middle to Lower Miocene strata in the southern part of Northeast Honshu Arc, Japan. Chishitsugaku Ronshu, 32:197-205. 
Tamaki, K., Pisciotto, K., Allan, J., et al., 1990. Proc. ODP, Init. Repts., 127: College Station, TX (Ocean Drilling Program).

Tanai, T., 1961. Neogene floral change of Japan. J. Fac. Sci. Hokkaido Univ.. Ser. 4, 11:119-398.

Traverse, A., and Ginsburg, R. N., 1966. Palynology of the surface sediments of Great Bahama Bank, as related to water movement and sedimentation. Mar. Geol., 4:417-459.

Yamanoi, T., 1976. Pollen analysis of Neogene sediments, the Tainai River Basin, Niigata Prefecture. Contrib. Dep. Geol. Mineral., Niigata Univ., 4:197-206.

, 1978a. Neogene pollen stratigraphy of the Oga Peninsula, Northeast Honshu, Japan. Chishitsugaku Zasshi, 84:69-86.

, 1978b. Neogene pollen stratigraphy of the Sado Island, Niigata Prefecture, Japan. Sekiyu Gijutsu Kyokaishi, 43:119-127.

, 1979. Neogene pollen stratigraphy of the Hachikoku district, Niigata Prefecture, Central Japan. Bull. Yamagata Univ., Sci., 9:613-628.

- 1983. Pollen stratigraphy of the Shinjo Group, Northeast Honshu, Japan. Mem. Nat. Sci. Mus., 16:37-52.

- 1984. Presence of sonneratiaceous pollen in Middle Miocene sediments, central Japan. Rev. Palaeobot. Palynol., 40:347-357.
1989. Neogene palynological zones and event in Japan. Proc. Int. Symp. Pacific Neogene Continental and Marine Events. Nanjing Univ. Press, IGCP-246:83-90.

, in press. The palyno-flora of Early Middle Miocene sediments in the Pohang and Yangnam Basins, Korea. Centenary of Japanese Micropaleontology.

Yamanoi, T., and Tsuda, K., 1986. On the condition of paleo-mangrove forest in the Kurosedani Formation (Middle Miocene), Central Japan. Mem. Nat. Sci. Mus., 19:55-66.

Yamanoi, T., Tsuda, K., Itoigawa, J., Okamoto, K., and Taguchi, E., 1980. On the mangrove community discovered from the Middle Miocene formations in Southwest Japan. Chishitsugaku Zasshi, 6:635-638.

Date of initial receipt: 21 March 1991

Date of acceptance: 23 September 1991

Ms 127/128B-150 


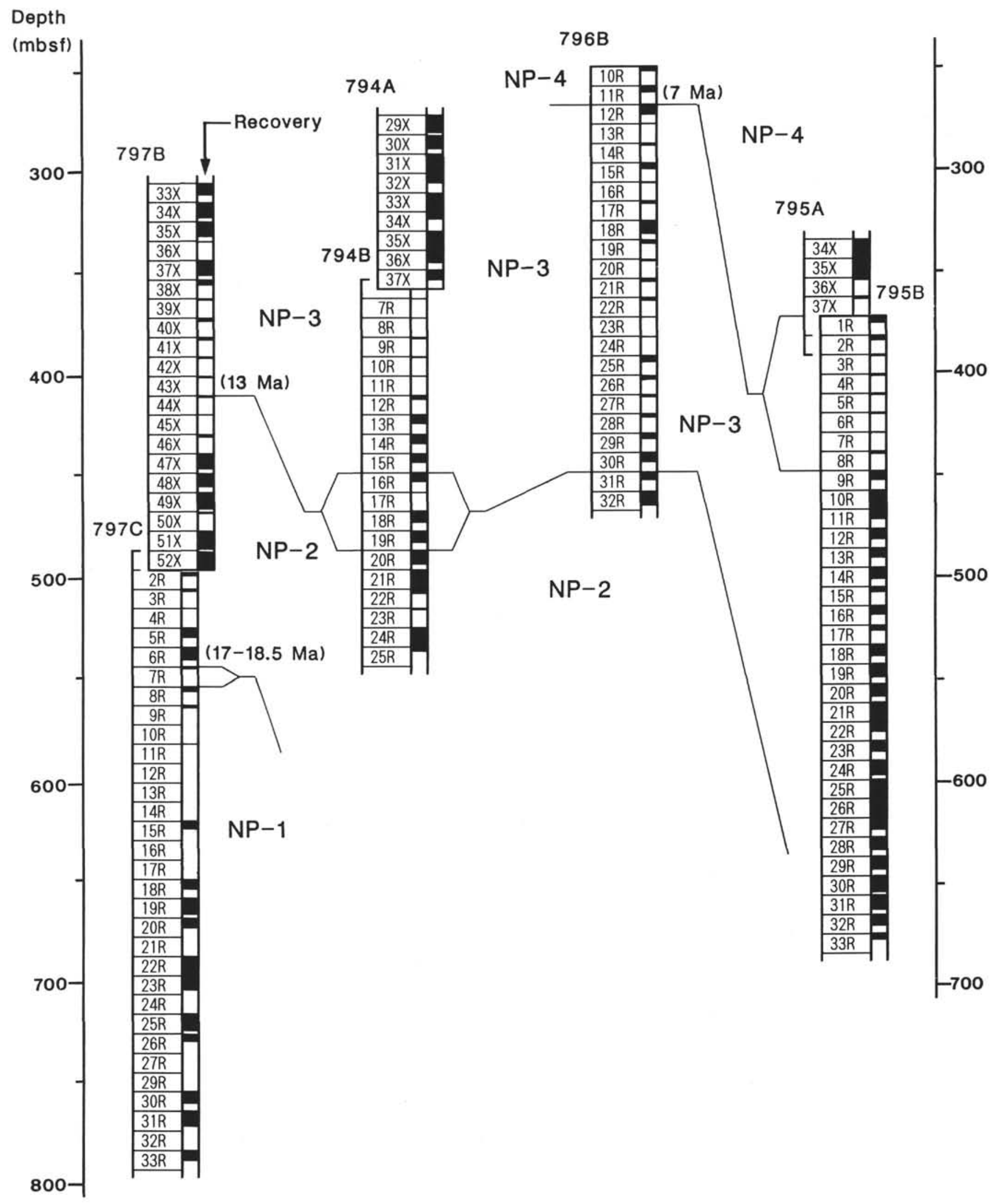

Figure 11. Correlation of Miocene pollen stratigraphies of four sites, Leg 127. 


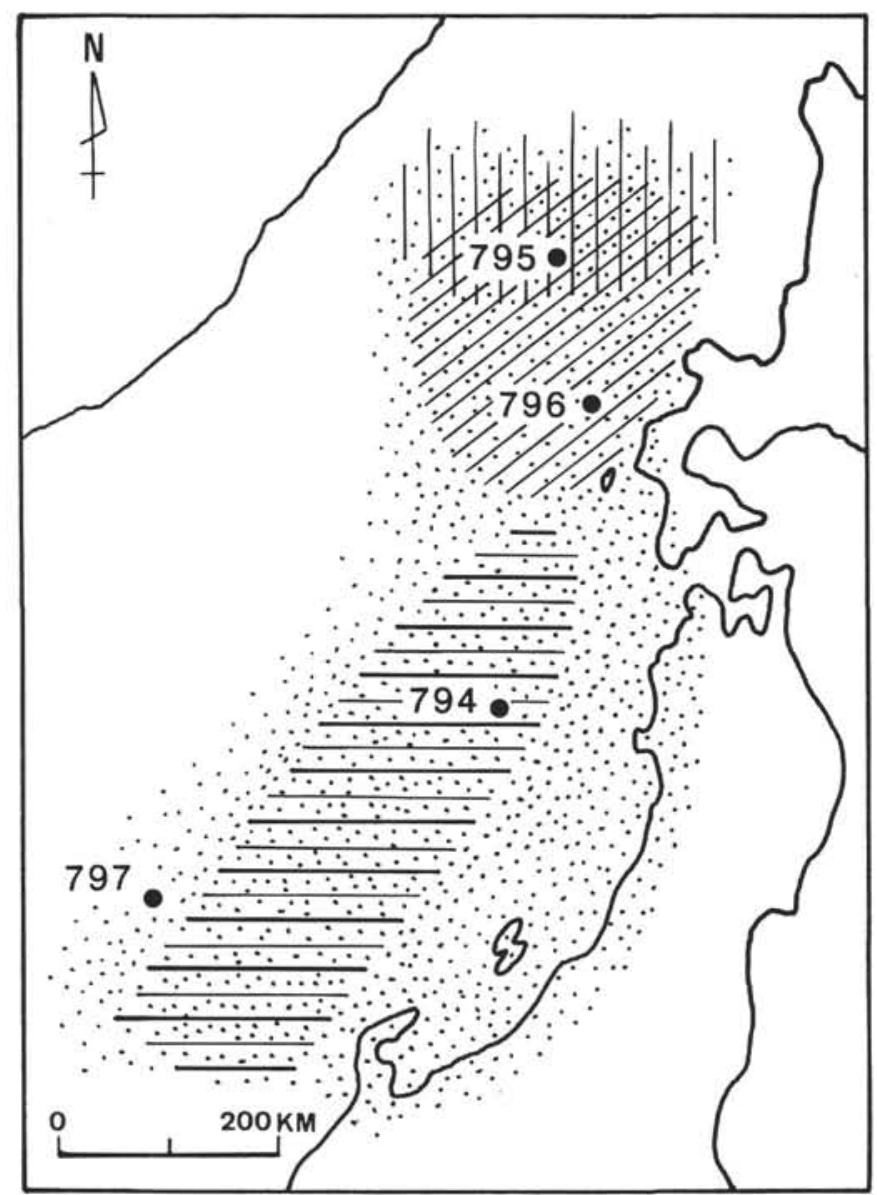

ए] Larix Z7axodiaceae

Figure 12. Map of the Japan Sea area showing the distribution of pollen fossils expected to occur abundantly in Zone NP3.

E. Quercus

Fagus +Carya

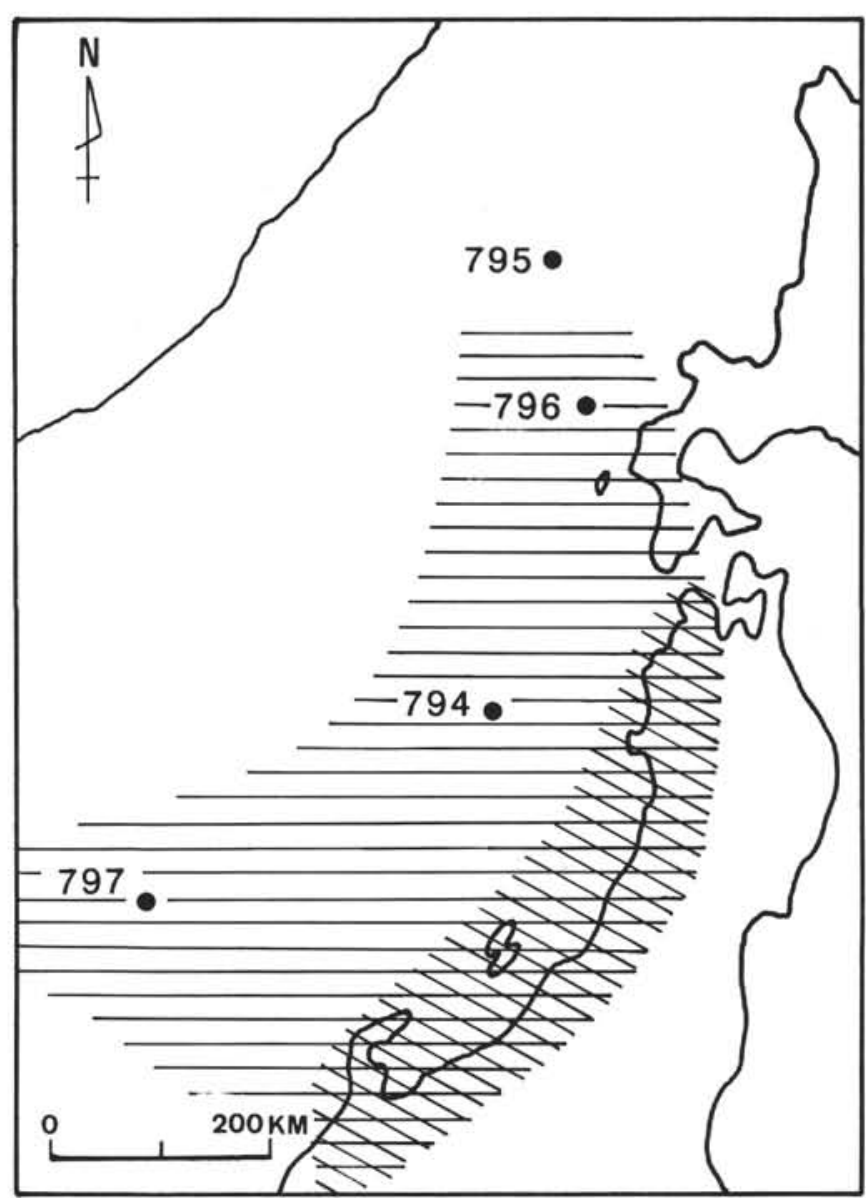

Figure 13. Map of the Japan Sea area showing the distribution of pollen fossils expected to occur abundantly in Zone NP2. 

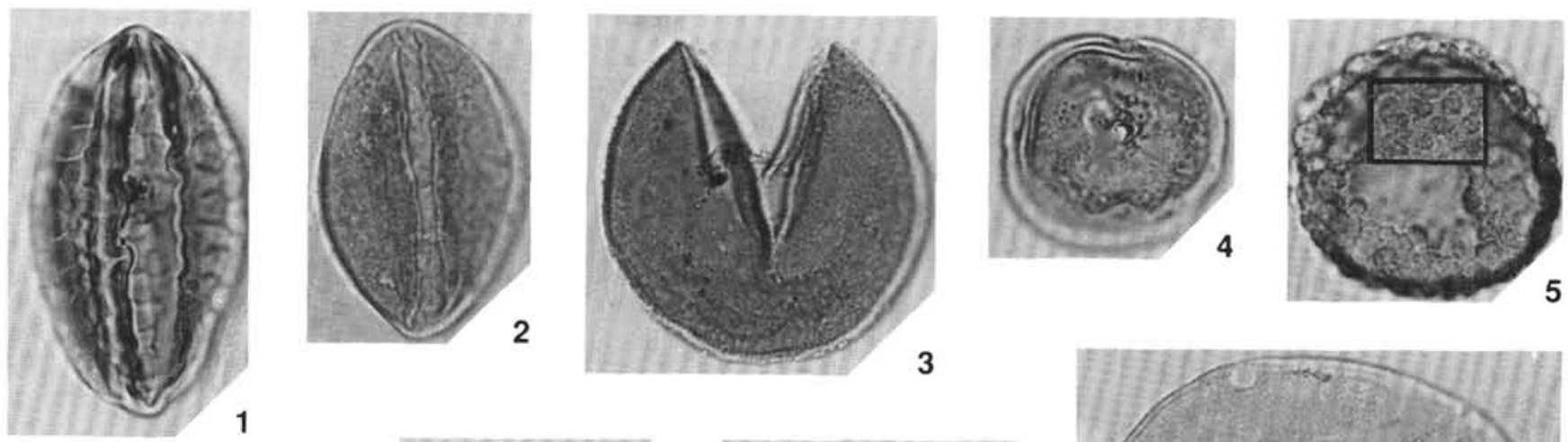

3

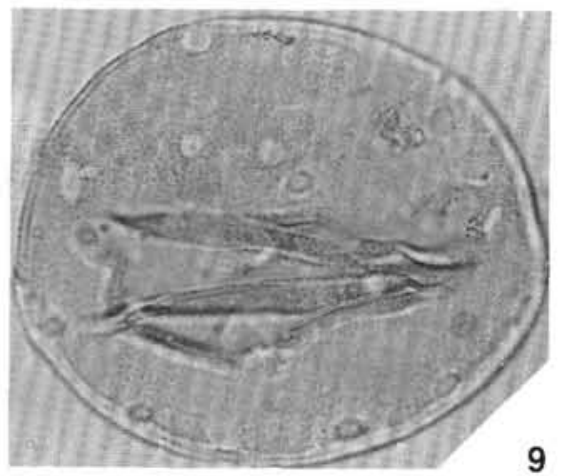

6
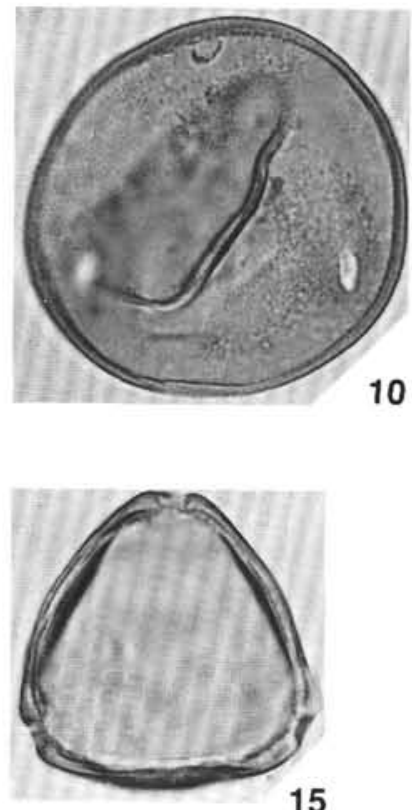

10

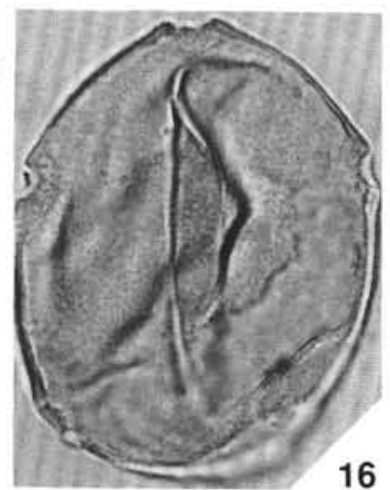

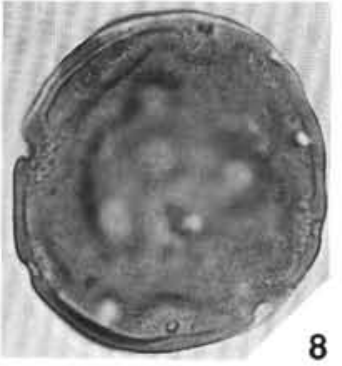
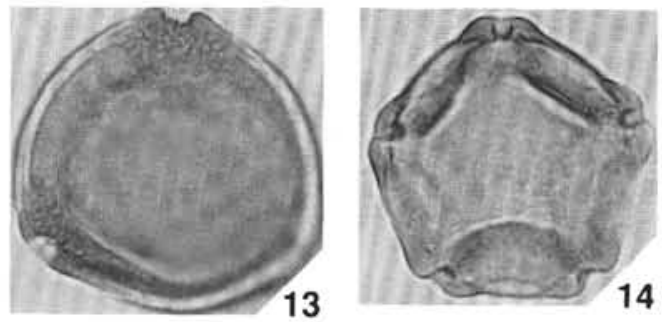

11
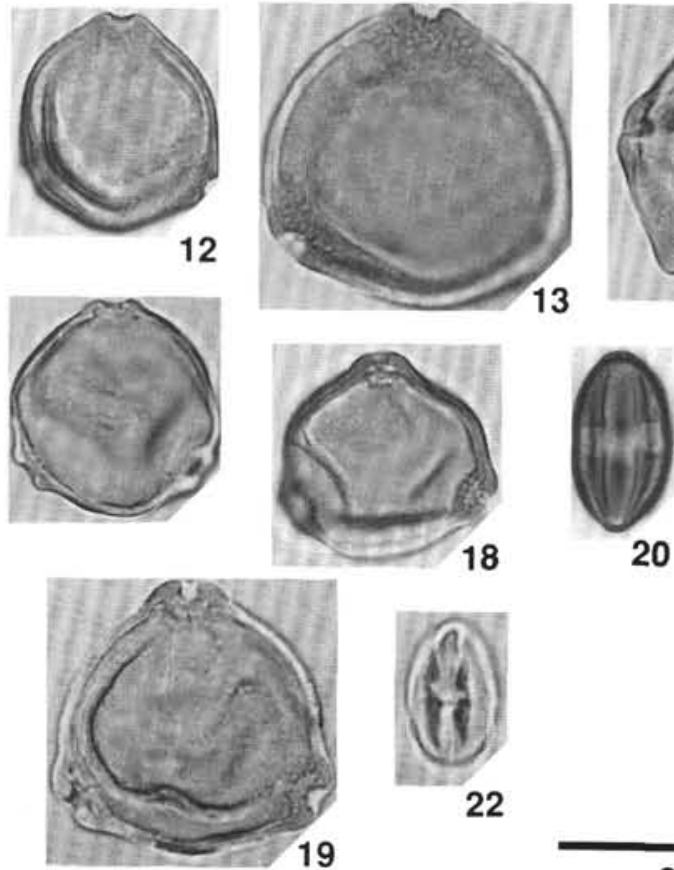

22

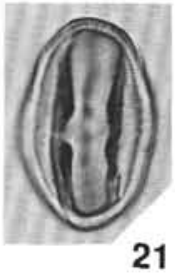

$30 \mu \mathrm{m}$

Plate 1. Pollen fossils of Leg 127 (LM). 1. Ephedra, Sample 127-795B-15R-2, 82-84 cm. 2. Ginkgo, Sample 127-795B-18R-2, 134-136 cm. 3. Taxodiaceae, Sample 127-795B-19R-2, 134-135 cm. 4. Metasequoia, Sample 127-796B-27R-1, 98-100 cm. 5. Sciadopitys, Sample 127-795B-15R-2, 82-84 cm. 6. Cunninghamia, Sample 127-797C-6R-4, 60-62 cm. 7. Cupressaceae, Sample 127-797C-8R-1,39-41 cm. 8. Juglans, Sample 127-795B-32R-3, 125-127 cm. 9. Juglans, Sample 127-794B-14R-2,71-72 cm. 10. Carya, Sample 127-795B-19R-2, 134-135 cm. 11. Pterocarya, Sample 127-794B-24R-1, 21-23 cm. 12. Engelhardia, Sample 127-796B-30R-1,83-85 cm. 13. Engelhardia, Sample 127-797B-43X-1,41-43 cm. 14. Alnus, Sample 127-795B-12R-2, 80-82 cm. 15. Corylus, Sample 127-794A-32X-1, 130-132 cm. 16. Carpinus, Sample 127-797B-43X-1, 41-43 cm. 17. Carpinus, Sample 127-797B-34X-5, 40-42 cm. 18. Betula, Sample 795B-15R-2, 82-84 cm. 19. Betula, Sample 127-797B-34X-5, 40-42 cm. 20. Pasania, Sample 127-794B-25R-CC, 13-15 cm. 21. Pasania, Sample 127-796B20R-1, 29-31 cm. 22. Castanea, Sample 127-794B-12R-2, 116-118 cm. 

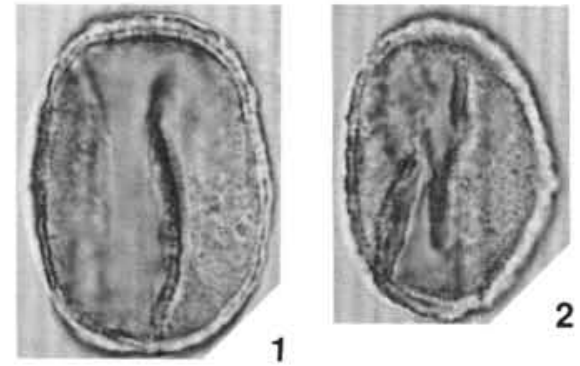

1
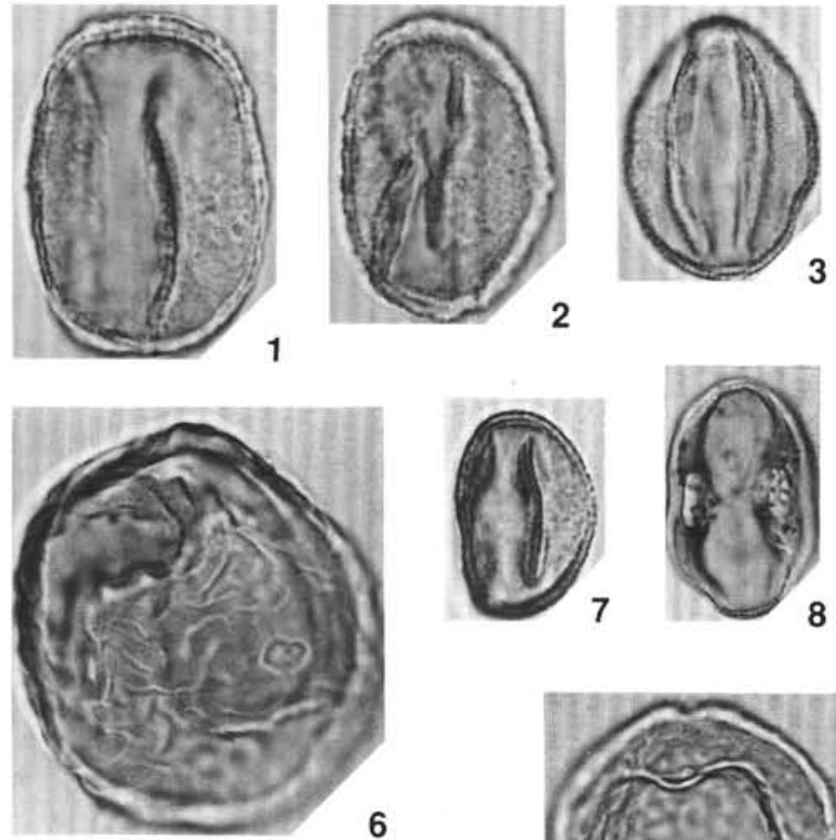

\section{2}

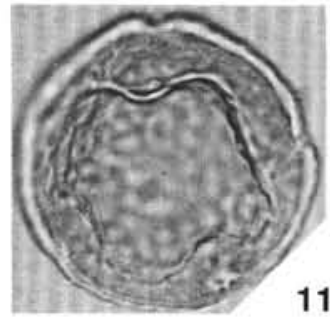

11
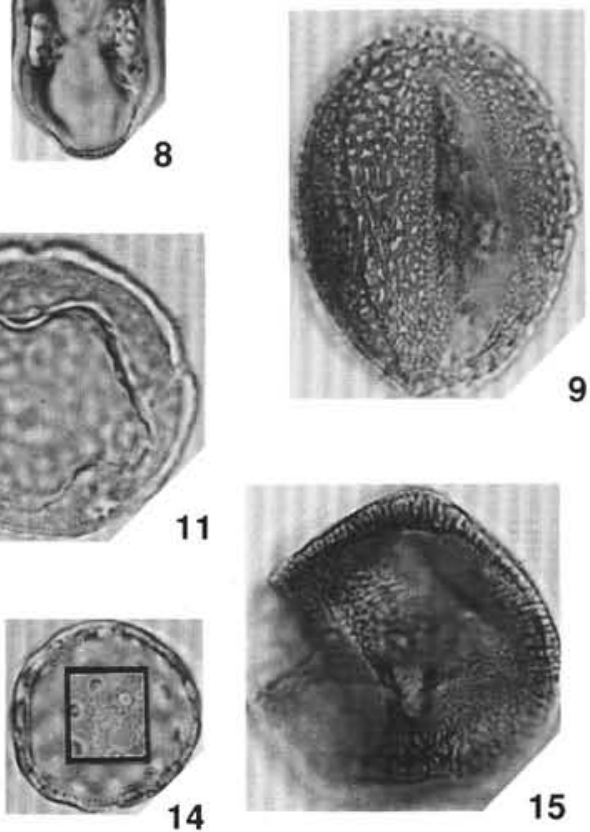

4
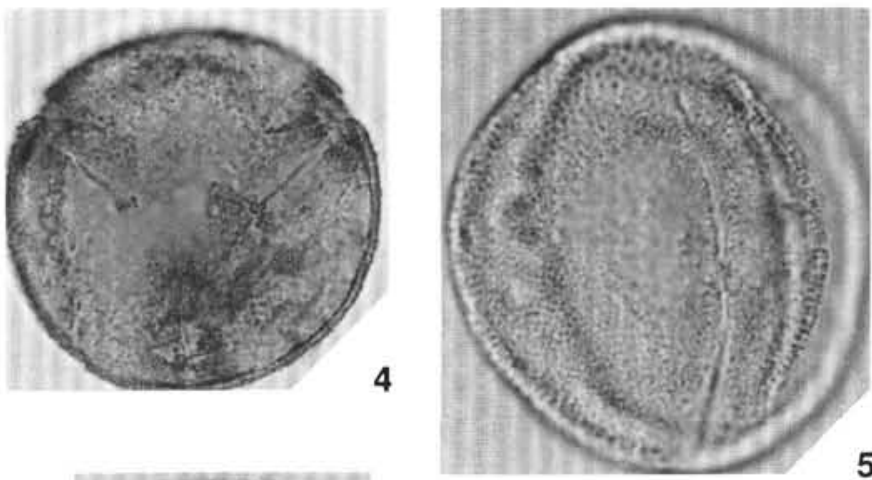

5
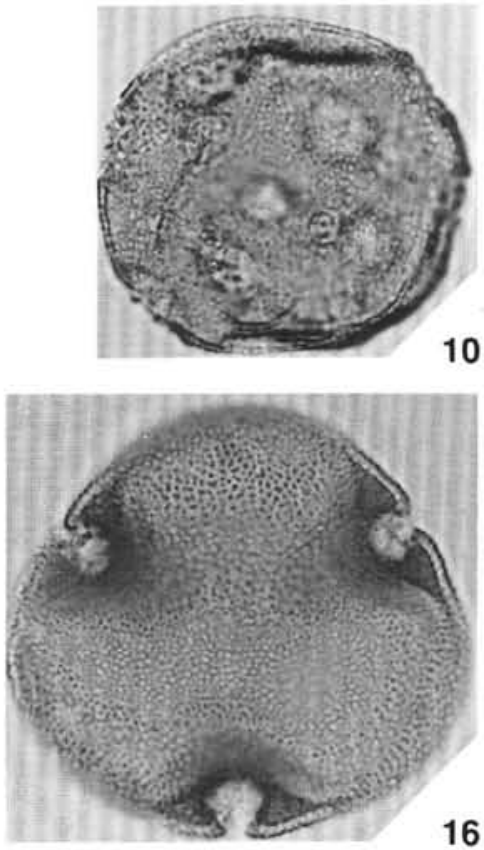
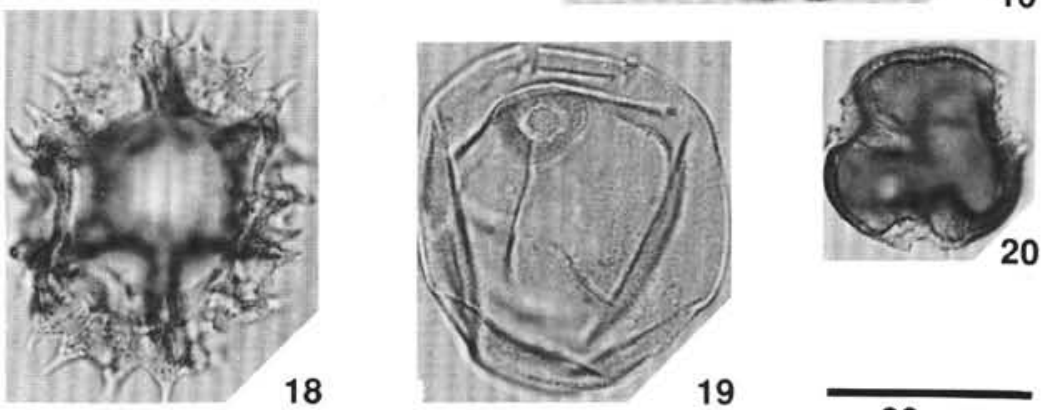

$20 \mu \mathrm{m}$

Plate 2. Pollen fossils of Leg 127 (LM) 1. D. (deciduous) Quercus, Sample 127-794A-35X-3, 117-119 cm. 2. D. Quercus, Sample 127-794A-35X-3, 117-119 cm. 3. E. (evergreen) Quercus, Sample 127-794A-36X-2, 39-41 cm. 4. Fagus, Sample 127-795B-21R-2, 132-134 cm. 5. Fagus, Sample 127-797B-34X-5, 40-42 cm. 6. Zelkoova, Sample 127-797B-43X-1, 41-43 cm. 7. E. Quercus, Sample 127-796B-19R-1, 133-135 cm. 8. Aesculus, Sample 127-796B-30IR-1, 83-85 cm. 9. Rhus, Sample 127-794B-25R-CC, 13-15 cm. 10. Liquidambar, Sample 127-797C-8R-1 39-41 cm. 11. Ulmus, Sample 127-795B-15R-2, 82-84 cm. 12. Artemisia, Sample 127-795B-12R-2, 80-82 cm. 13. Artemisia, Sample 127-795B-12R-2, 80-82 cm. 14. Chenopodiaceae, Sample 127-794A-37X-2, 127-129 cm. 15. Fupingopollenites, Sample 127-797C-8R-1, 39-41 cm. 16. Tilia, Sample 127-797B-42X-1, 40-42 cm. 17. Carduoideae, Sample 127-795A-32X-2, 135-136 cm. 18. Cichorioideae, Sample 127-795B-14B-1, 87-89 cm. 19. Gramineae, Sample 127-794A$33 \mathrm{X}-3,130-132 \mathrm{~cm}$. 20. Eurya, Sample 127-796B-3R-2, 88-90 cm. 